\title{
Optical Diffraction Radiation from a beam off a circular target
}

\author{
Tanaji Sen \\ Accelerator Physics Center, FNAL \\ PO Box 500 \\ Batavia, IL 60510
}

\section{Contents}

1 Introduction $\quad 2$

2 Hadron colliders $\quad 3$

3 ODR from a round hole $\quad 3$

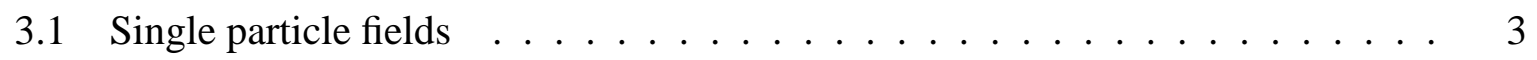

3.1.1 Particle at the center of target . . . . . . . . . . . . 4

3.1.2 Particle offset from the center of target . . . . . . . . . . . . . 10

3.2 Spectrum from a bunch . . . . . . . . . . . . . . 16

4 Sensitivity to beam parameters 17

5 Photon yield $\quad 20$

6 OTR vs ODR spectrum 26

7 Experimental Issues $\quad 27$

8 Conclusions $\quad 28$

\begin{abstract}
We calculate the optical diffraction radiation generated by a bunch of high energy particles as they pass through a round hole within an annular metallic ring. We derive expressions for the differential angular spectrum in the far-field and the intensities of the horizontal and vertical polarizations. The sensitivity of the spectrum to changes in beam size and position is shown. The total photon yield from the bunch is calculated and used to set limits on the detectable wavelengths.
\end{abstract}




\section{Introduction}

The use of optical diffraction radiation (ODR) as a diagnostic tool has increased in recent years. The potential of this technique has been demonstrated in several experiments at KEK [1], APS [2], FLASH [3] and possibly other facilities. These experiments were performed in extraction beam lines of lepton machines. However this technique can also be applied to high energy hadron beams. In this report we consider the ODR produced by such beams with the target as a round hole and apply the results to the Tevatron.

This radiation is produced when a beam passes in the vicinity of a conducting target. The electro-magnetic fields due to the beam induce currents on the target and as the beam propagates, the currents change in time producing radiation both in the direction of beam propagation and along the direction of specular reflection from the target. This latter radiation, also termed backward diffraction radiation (BDR), is more useful for diagnostics since it can be directed out at the same longitudinal location as the target. This radiation is different from optical transition radiation (OTR) in which the beam passes through a metal target. Transition radiation is not suitable for continuous monitoring of a beam in a collider due to the beam energy loss and emittance growth and the fact that the target may be damaged. However the techniques for analyzing ODR are similar in many respects to those for OTR.

Measurements of the radiation intensity either in the near field or far-field have been used to determine beam positions and sizes. For example, the beam size and beam position of a 1.28 $\mathrm{GeV}$ electron beam were measured in an extraction beam line at KEK [1] using the far-field angular distribution of the radiation. The near-field image was used to monitor the relative beam size of a $7 \mathrm{GeV}$ electron beam in the extraction line at APS [2]. In principle, measurements of the beam divergence are also possible using the interference of ODR between two targets, as has been done with OTR.

This paper is motivated by the desire to use this technique in colliders, especially for the LHC and possibly for future colliders envisaged such as the muon collider. A brief report on these prospects was presented earlier [4]. If the technique yields beam measurements with sufficient accuracy and reliability then the non-invasive nature would allow continuous monitoring during the length of a luminosity run. This would be valuable if the beam can be imaged close to the interaction points.

Synchrotron radiation is already used as a non-invasive diagnostic tool in the Tevatron and will also be used in the LHC. The principal advantage of ODR is that it can be generated in a straight section and therefore used for imaging in an experimental insertion. The disadvantage is that the ODR flux is less copious than synchrotron radiation (OSR) and imaging will take longer than with OSR.

In Section 2 we briefly discuss the parameters of different hadron colliders. In Section 3 we derive the basic results for the angular differential spectrum of ODR from a round hole due to a bunch. We apply these results in Section 4 to find the sensitivity of the spectrum to beam size and offset changes. In Section 5, we calculate the expected photon yield from a bunch per turn as a function of frequency and we use this to find the frequency range where a sufficiently strong ODR signal can be obtained. In Section 6 we do a brief comparison of the ODR spectrum with the OTR spectrum. We briefly list in Section 7 the experimental issues 


\begin{tabular}{|c|c|c|c|}
\hline & Tevatron & RHIC & LHC \\
\hline Energy [GeV] & 980 & 250 & 7000 \\
Bunch intensity & $2.7 \times 10^{11}$ & $2 \times 10^{11}$ & $1.1 \times 10^{11}$ \\
Beam size $[\mu \mathrm{m}]$ & 400 & 1012 & 807 \\
Beam div/opening angle & $2.9 \times 10^{-3}$ & $1.2 \times 10^{-5}$ & $5.7 \times 10^{-3}$ \\
Number of bunches & 36 & $55 \rightarrow 120$ & 2808 \\
Revolution frequency $[\mathrm{kHz}]$ & 47.6 & 78.2 & 11.2 \\
\hline
\end{tabular}

Table 1: Table of parameters for hadron colliders

associated with measuring ODR when two beams are present. We end with our conclusions in Section 8. We will use CGS units throughout.

\section{Hadron colliders}

Optical transition radiation (OTR) has been used in the Tevatron at injection energy to image the beam [6]. At collision this technique is not feasible both because of the impact on beam quality via multiple scattering in the target and the damage to the target itself. However ODR is non-intercepting and has the potential to be a useful diagnostic tool at collision energy. This technique also has potential in the LHC where we envision that placing ODR targets on both sides of the interaction point (IP) and before the first interaction region quadrupole would allow a non-invasive measurement of the beam size at the IP. At RHIC the energy is lower so one would have to use longer wavelength ODR for a substantial radiation flux.

Table 1 shows some of the key parameters for these hadron colliders. The beam size in the Tevatron was calculated at $\mathrm{C} 0$ while for RHIC and the $\mathrm{LHC}$, locations in front of the first interaction region quadrupole were chosen. The ratio of the beam divergence to the opening angle of the radiation $(\sim 1 / \gamma)$ is very small in all the colliders, hence the distortion of the spectrum due to the beam divergence should be negligible.

\section{ODR from a round hole}

The fields induced by a beam as it passes through a hole depends on the beam energy, the beam size, the beam position relative to the center of the hole and the shape of the hole. In this paper we consider a round hole as a target. First we analyse the fields from a single particle and generalize results obtained many years ago by Ter-Mikaelian [5]. Next we consider the ODR fields and spectrum generated by a Gaussian bunch of particles.

\subsection{Single particle fields}

Consider the case where a single particle moving at constant velocity $v$ goes through a round annulus made of conducting material with inner and outer radii of $a_{\text {in }}$ and $a_{\text {out }}$ respectively. 
The fields of the particle induce fields on the surface of the annulus. We introduce the Fourier transform of the fields as

$$
E_{x}=\int E_{\omega, x} e^{-i \omega t} d \omega \quad E_{y}=\int E_{\omega, y} e^{-i \omega t} d \omega
$$

The Fourier transformed transverse fields of a particle moving at constant velocity along the $z$ axis are given by [5]

$$
\begin{aligned}
& E_{\omega, x}=\frac{q \alpha}{\pi v} \frac{x}{\rho} e^{i \omega z / v} K_{1}(\alpha \rho) \\
& E_{\omega, y}=\frac{q \alpha}{\pi v} \frac{y}{\rho} e^{i \omega z / v} K_{1}(\alpha \rho)
\end{aligned}
$$

The origin of coordinates is at the center of the hole and in particular the hole is in the $z=0$ plane. $q$ is the particle charge, and

$$
\alpha=\frac{\omega}{v \gamma}=\frac{k}{\gamma}, \quad \rho=\left(x^{2}+y^{2}\right)^{1 / 2}
$$

$K_{1}$ is a modified Bessel function of order one.

\subsubsection{Particle at the center of target}

We will first consider the simpler case of the particle moving the center of the target. We will derive equations for the fields and angular spectral distribution which will serve as a useful check of the more general case when the particle is offset from the center of the hole.

Consider the field at any arbitrary point on the surface of the hole. Given the axial symmetry of the target, we use polar coordinates. The coordinates of a point $(x, y)$ on the target are

$$
x=\rho \cos \phi, \quad y=\rho \sin \phi
$$

Then

$$
\left[\begin{array}{c}
E_{\omega, x} \\
E_{\omega, y}
\end{array}\right]=\frac{q \alpha}{\pi v} K_{1}(\alpha \rho)\left[\begin{array}{c}
\cos \phi \\
\sin \phi
\end{array}\right]
$$

We will calculate the fields at an arbitrary location using scalar diffraction theory. Within this approximation of assuming scalar diffraction theory to be valid, the fields from the entire target at an arbitrary observation point $\mathrm{P}$ can be found by integrating over the annulus

$$
\left[\begin{array}{c}
E_{\omega, x} \\
E_{\omega, y}
\end{array}\right]=-\frac{i k}{2 \pi} \frac{q \alpha}{\pi v} \int_{a_{\text {in }}}^{a_{\text {out }}} \rho d \rho \int_{0}^{2 \pi} d \phi \frac{e^{i k R^{\prime}}}{R^{\prime}} K_{1}(\alpha \rho)\left[\begin{array}{c}
\cos \phi \\
\sin \phi
\end{array}\right]
$$

where $R^{\prime}$ is the distance from the point on the target to the observation point $\mathrm{P}$. If $(x, y, z)$ are the coordinates of $\mathrm{P}$, then

$$
R^{\prime}=\left[(x-\rho \cos \phi)^{2}+(y-\rho \sin \phi)^{2}+z^{2}\right]^{1 / 2}
$$


Far field spectrum

The observation point $\mathrm{P}$ is assumed to be sufficiently far from the target so that all points on the target have nearly the same phase from P. In this case the linear dimensions of the target are small compared to the distance from the target. This is the regime of Fraunhoffer diffraction.

If $R$ is the distance from the center of the hole to the point P, i.e. $R=\left[x^{2}+y^{2}+z^{2}\right]^{1 / 2}$, then we assume here that $a_{i n}, a_{\text {out }} \ll R$. Thus in the phase term $e^{i k R^{\prime}}$ we expand

$$
R^{\prime} \simeq R\left[1-\frac{2 \rho}{R^{2}}(x \cos \phi+y \sin \phi)\right]^{1 / 2} \simeq R-\rho \sin \theta_{P} \cos \left(\phi_{P}-\phi\right)
$$

where we define

$$
x=\rho_{P} \cos \phi_{P}, \quad y=\rho_{P} \sin \phi_{P}, \quad \sin \theta_{P}=\frac{\rho_{P}}{R}, \quad \bar{k}=k \sin \theta_{P}
$$

Then

$$
\left[\begin{array}{c}
E_{\omega, x} \\
E_{\omega, y}
\end{array}\right]=-\frac{i k}{2 \pi} \frac{q \alpha}{\pi v} \frac{e^{i k R}}{R} \int_{a_{\text {in }}}^{a_{\text {out }}} \rho d \rho \int_{0}^{2 \pi} d \phi K_{1}(\alpha \rho) \exp \left[-i \bar{k} \rho \cos \left(\phi_{P}-\phi\right)\right]\left[\begin{array}{c}
\cos \phi \\
\sin \phi
\end{array}\right]
$$

To do the $\phi$ integrals, we use the integral representation of the integer Bessel functions

$$
J_{n}(z)=\frac{i^{n}}{2 \pi} \int_{0}^{2 \pi} \exp [-i z \cos \phi] \cos n \phi d \phi
$$

Then

$$
\int_{0}^{2 \pi} \exp \left[-i \bar{k} \rho \cos \left(\phi_{P}-\phi\right)\right]\left[\begin{array}{c}
\cos \phi \\
\sin \phi
\end{array}\right]=\frac{2 \pi}{i} J_{1}(\bar{k} \rho)\left[\begin{array}{c}
\cos \phi_{P} \\
\sin \phi_{P}
\end{array}\right]
$$

The integral over the radius yields

$$
\begin{aligned}
\int_{a_{\text {in }}}^{a_{\text {out }}} \rho d \rho K_{1}(\alpha \rho) J_{1}(\bar{k} \rho)= & \frac{1}{\bar{k}^{2}+\alpha^{2}}\left\{a_{\text {out }}\left[\bar{k} J_{2}\left(\bar{k} a_{\text {out }}\right) K_{1}\left(\alpha a_{\text {out }}\right)-\alpha J_{1}\left(\bar{k} a_{\text {out }}\right) K_{2}\left(\alpha a_{\text {out }}\right)\right]\right. \\
& \left.-a_{\text {in }}\left[\bar{k} J_{2}\left(\bar{k} a_{\text {in }}\right) K_{1}\left(\alpha a_{\text {in }}\right)-\alpha J_{1}\left(\bar{k} a_{\text {in }}\right) K_{2}\left(\alpha a_{\text {in }}\right)\right]\right\}
\end{aligned}
$$

Using the recurrence relations

$$
x J_{n+1}(x)=2 n J_{n}(x)-x J_{n-1}(x), x K_{n+1}(x)=2 n K_{n}(x)-x K_{n-1}(x)
$$

we can write

$$
\int_{a_{\text {in }}}^{a_{\text {out }}} \rho d \rho K_{1}(\alpha \rho) J_{1}(\bar{k} \rho)=\frac{1}{\bar{k}^{2}+\alpha^{2}}\left[T\left(a_{\text {out }} ; \bar{k}\right)-T\left(a_{\text {in }} ; \bar{k}\right)\right]
$$

where

$$
T(a ; \bar{k})=-a\left[\bar{k} J_{0}(\bar{k} a) K_{1}(\alpha a)+\alpha J_{1}(\bar{k} a) K_{0}(\alpha a)\right]
$$

Thus the fields are

$$
\left[\begin{array}{c}
E_{\omega, x} \\
E_{\omega, y}
\end{array}\right]=-\frac{k q \alpha}{\pi v} \frac{e^{i k R}}{R} \frac{1}{\bar{k}^{2}+\alpha^{2}}\left[T\left(a_{\text {out }} ; \bar{k}\right)-T\left(a_{i n} ; \bar{k}\right)\right]\left[\begin{array}{c}
\cos \phi_{P} \\
\sin \phi_{P}
\end{array}\right]
$$


The Poynting vector is

$$
\vec{S}=\frac{c}{4 \pi} \vec{E} \times \vec{B}^{*}=\frac{c}{4 \pi}\left[-\beta E_{z} E_{x}^{*} \hat{x}-\beta E_{z} E_{y}^{*} \hat{y}+\beta\left(\left|E_{x}\right|^{2}+\left|E_{y}\right|^{2}\right) \hat{z}\right]
$$

where we have used

$$
B_{x}=-\beta E_{y}, \quad B_{y}=\beta E_{x}, \quad B_{z}=0
$$

The total energy deposited by the fields onto an element of area $d A$ is the time integral of the projecteg Poynting vector

$$
\frac{d W}{d A}=\int_{-\infty}^{\infty} d t \vec{S} \cdot \hat{n}
$$

where $\hat{n}$ is the unit normal to the element. For an element orthogonal to the direction of propagation or direction of specular reflection

$$
\frac{d W}{d A}=\int_{-\infty}^{\infty} d t S_{z}=\frac{\beta c}{4 \pi} \int_{-\infty}^{\infty} d t\left(\left|E_{x}\right|^{2}+\left|E_{y}\right|^{2}\right)=2 \pi \int d \omega\left[\left|E_{\omega, x}\right|^{2}+\left|E_{\omega, y}\right|^{2}\right]
$$

Hence the differential angular spectrum is

$$
\frac{d^{2} W}{d \Omega d \omega}=\frac{1}{2} \beta c R^{2}\left[\left|E_{\omega, x}\right|^{2}+\left|E_{\omega, y}\right|^{2}\right]
$$

where $d \Omega$ is the solid angle subtended by the element at a distance $R$ from the source.

Thus in the far field, the differential spectrum is

$$
\frac{d^{2} W}{d \Omega d \omega}=\frac{1}{2} \beta c\left(\frac{k q \alpha}{\pi v}\right)^{2} \frac{1}{\left[\bar{k}^{2}+\alpha^{2}\right]^{2}}\left[T\left(a_{\text {out }} ; \bar{k}\right)-T\left(a_{\text {in }} ; \bar{k}\right)\right]^{2}
$$

Define a critical frequency $\omega_{c}$, and dimensionless parameters $u, t, g$ as

$$
\omega_{c}=\frac{\gamma_{c}}{a_{\text {in }}}, \quad u=\frac{\omega}{\omega_{c}}, \quad t=\gamma \sin \theta_{p}, \quad g=\frac{a_{\text {out }}}{a_{\text {in }}}
$$

Then other parameters can be written in terms of these dimensionless parameters as $k=\gamma u /\left(\beta a_{i n}\right)$, $\alpha=u /\left(\beta a_{i n}\right)$ etc and

$$
\begin{aligned}
{\left[T\left(a_{\text {out }} ; \bar{k}\right)-T\left(a_{\text {in }} ; \bar{k}\right)\right]^{2}=} & \left(\frac{u}{\beta}\right)^{2}\left\{g\left[t J_{0}\left(\frac{1}{\beta} g u t\right) K_{1}\left(\frac{1}{\beta} g u\right)+J_{1}\left(\frac{1}{\beta} g u t\right) K_{0}\left(\frac{1}{\beta} g u\right)\right]\right. \\
& \left.-\left[t J_{0}\left(\frac{1}{\beta} u t\right) K_{1}\left(\frac{1}{\beta} u\right)+J_{1}\left(\frac{1}{\beta} u t\right) K_{0}\left(\frac{1}{\beta} u\right)\right]\right\}
\end{aligned}
$$

The angular spectral distribution thus is

$$
\begin{aligned}
\frac{d^{2} W}{d \Omega d \omega}= & \frac{\beta c}{2}\left(\frac{q \gamma}{\pi v}\right)^{2} \frac{u^{2}}{\left[1+t^{2}\right]^{2}}\left\{g\left[t J_{0}\left(\frac{1}{\beta} g u t\right) K_{1}\left(\frac{1}{\beta} g u\right)+J_{1}\left(\frac{1}{\beta} g u t\right) K_{0}\left(\frac{1}{\beta} g u\right)\right]\right. \\
& \left.-\left[t J_{0}\left(\frac{1}{\beta} u t\right) K_{1}\left(\frac{1}{\beta} u\right)+J_{1}\left(\frac{1}{\beta} u t\right) K_{0}\left(\frac{1}{\beta} u\right)\right]\right\}^{2}
\end{aligned}
$$

We comment on some features of this expression 
- This spectrum depends on the magnitude of the inner radius $a_{i n}$ only through the critical frequency $\omega_{c}$.

- The main dependence of the spectrum on the size of the target is through the dimensionless ratio $g=a_{\text {out }} / a_{i n}$. This is important since it suggests that the target hole may be enlarged to allow more space for the beam while at the same time increasing the outer radius without changing the spectrum. The parameter that will change in this case is the critical frequency $\omega_{c}$ and consequently the dimensional frequency $\omega$.

Th differential spectrum may be found by integrating over the solid angle

$$
\frac{d W}{d \omega}=\int \frac{d^{2} W}{d \Omega d \omega} d \phi \sin \theta_{P} d \theta_{P}=\frac{4 \pi}{\gamma^{2}} \int_{0}^{\gamma} \frac{d^{2} W}{d \Omega d \omega} \frac{t}{\sqrt{1-t^{2} / \gamma^{2}}} d t
$$

Define the function

$$
\begin{aligned}
F(g, u)= & \int_{0}^{\gamma} d t \frac{t}{\sqrt{1-t^{2} / \gamma^{2}}} \frac{1}{\left[1+t^{2}\right]^{2}}\left\{g\left[t J_{0}\left(\frac{1}{\beta} g u t\right) K_{1}\left(\frac{1}{\beta} g u\right)+J_{1}\left(\frac{1}{\beta} g u t\right) K_{0}\left(\frac{1}{\beta} g u\right)\right]\right. \\
& \left.-\left[t J_{0}\left(\frac{1}{\beta} u t\right) K_{1}\left(\frac{1}{\beta} u\right)+J_{1}\left(\frac{1}{\beta} u t\right) K_{0}\left(\frac{1}{\beta} u\right)\right]\right\}^{2}
\end{aligned}
$$

The number of photons $\Delta N$ emitted by a single charged particle into a bandwidth $\Delta \omega$ is

$$
\Delta N \equiv \frac{d N}{d \omega} \Delta \omega=\left(\frac{1}{\hbar \omega} \frac{d W}{d \omega}\right) \Delta \omega
$$

which can be written as

$$
\Delta N_{p h}=\left(\frac{1}{\pi \beta} \alpha_{f}\right) u^{2} F(g, u) \frac{\Delta \omega}{\omega}
$$

where $\alpha_{f}=q^{2} /(\hbar c) \approx 1 / 137$ is the fine structure constant. This depends on the relative frequency $u=\omega / \omega_{c}$ and the relative bandwidth $\Delta \omega / \omega$.

\section{Example: Tevatron parameters}

We evaluate the spectrum and the number of photons per particle for the Tevatron. Energy $=980 \mathrm{GeV}$, number of particles per bunch $N_{p}=2.7 \times 10^{11}$. Figure 1 shows the differential angular spectrum as a function of the parameters $g, t$ with the frequency fixed at $\omega=\omega_{c}$. Figure 2 shows the spectrum as a function of the parameters $u, t$ at a fixed ratio $g=1.5$. See the figure captions for comments.

Figure 3 shows the number of photons per particle calculated using the expression Eq (24) and assuming $\omega=\omega_{c}$ and a $1 \%$ bandwidth or $\Delta \omega / \omega=0.01$. The curve again shows that there is little gain in intensity when the target size increases beyond $g>2.5$. After some transverse distance from the particle, its field has dropped to sufficiently low values that no radiators in the target can be excited and therefore there is no further increase in the ODR radiation with increasing material in the target. The number of photons emitted by a single particle in one pass 


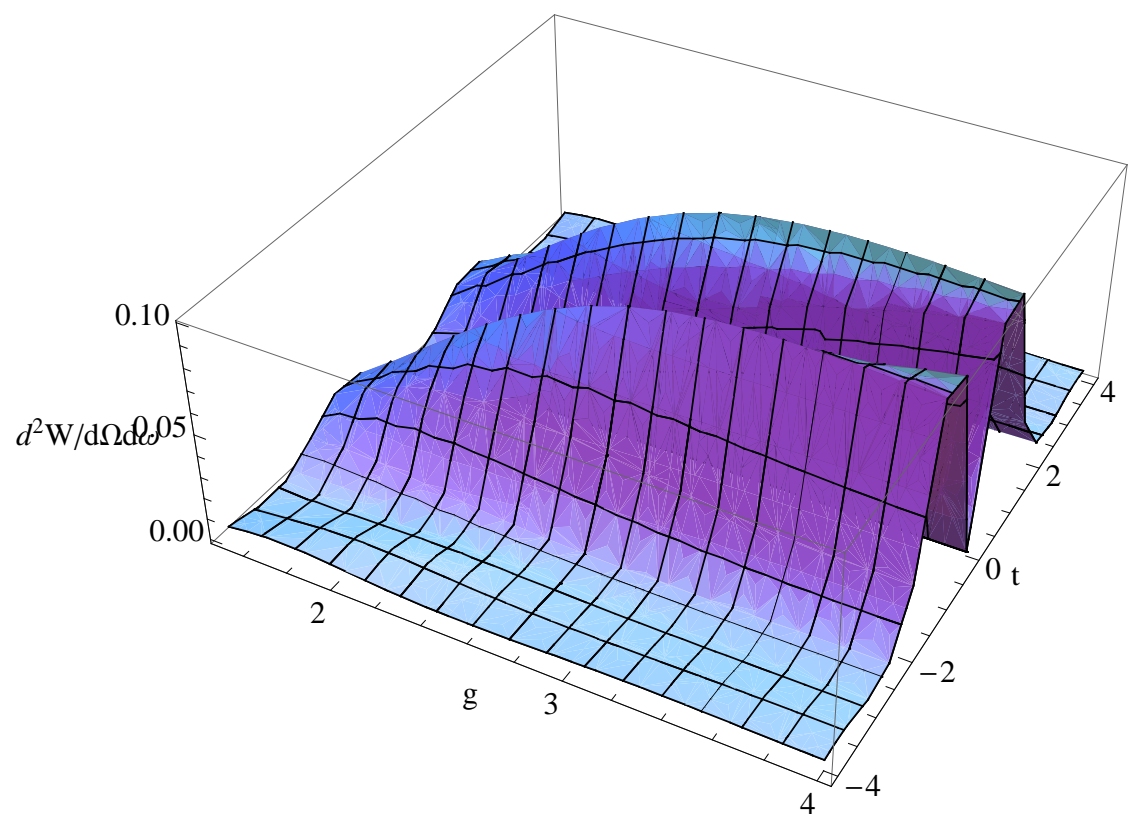

Figure 1: The differential angular spectrum as a function of the ratio of the outer and inner radii of the target $g=a_{\text {out }} / a_{\text {in }}$ and the angular variable $t=\gamma \sin \theta_{P}$ at constant $u=1$. Note that the spectrum saturates as a function of $g$ for $g>3$.

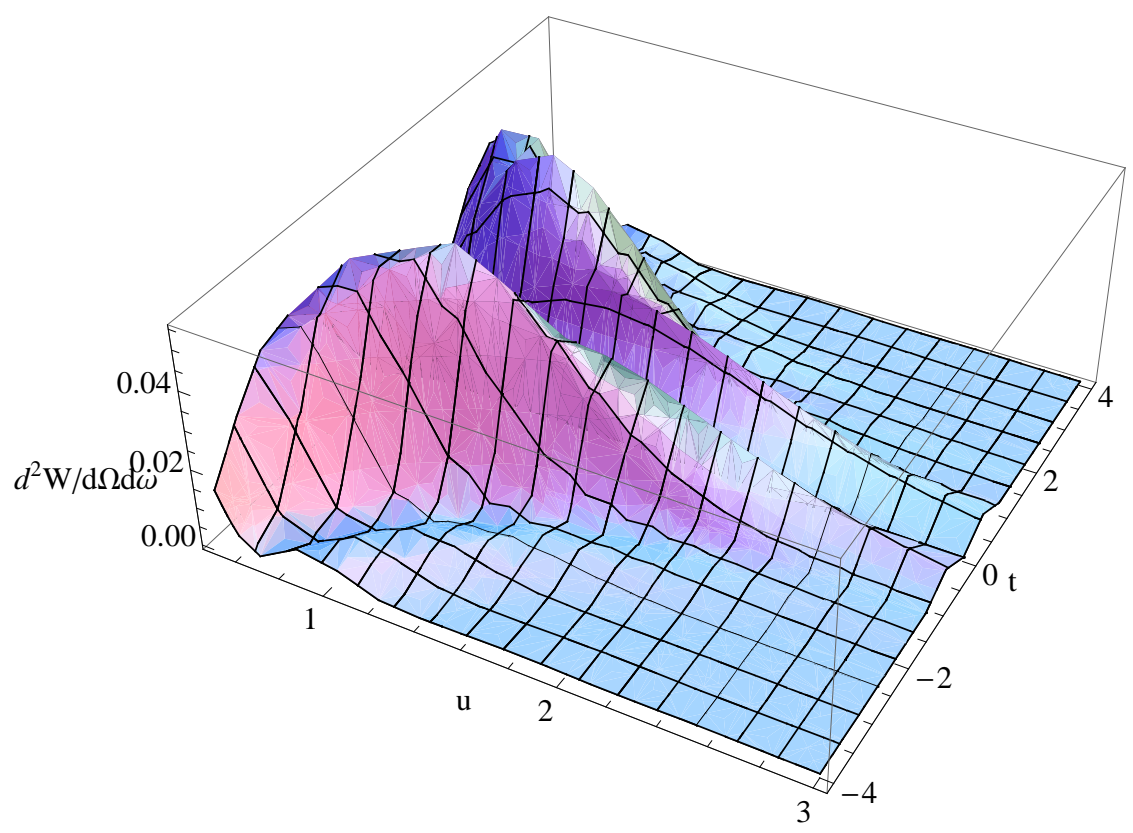

Figure 2: The differential angular spectrum as a function of the ratio $u=\omega / \omega_{c}$ and the angular variable $t=\gamma \sin \theta_{P}$ at constant $g=1.5$. The spectrum peaks close to $u=1$, i.e. close to the critical frequency $\omega_{c}$. 


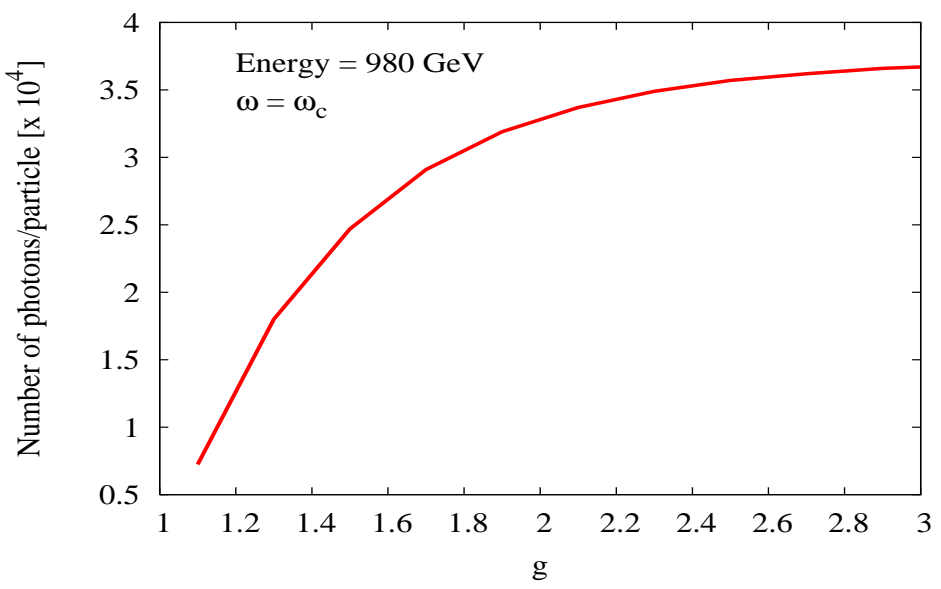

Figure 3: The number of photons per particle as a function of the parameter $g$ emitted at the critical frequency $\omega_{c}$. We observe that for $g>2$, the number of photons increases slowly with $g$.

through the center of the target can be found from this curve which is calculated for the critical frequency $\omega_{c}$. For example at

$$
g=1.1 \Rightarrow \Delta N=1.44 \times 10^{-4}
$$

A very simple estimate for the number of photons emitted by a bunch in a single pass at the frequency $\omega_{c}$ is therefore

$$
\Delta N(\text { bunch })=N_{p} \times \Delta N=3.2 \times 10^{5}
$$

This in fact is an underestimate since it assumes that all particles are at the center of the target and therefore furthest from the material of the target. A more precise estimate using the density distribution of the bunch will be obtained in the following section.

Near field spectrum

Here we calculate the field distribution at a distance close enough to the target that the phase differences between different points on the target to the observation point is significant. This is the region of Fresnel diffraction.

Here in the expansion for $R^{\prime}$ we keep the next order term in $\rho / R$. Thus

$$
R^{\prime}=R\left[1-2 \frac{\rho \rho_{P}}{R 62} \cos \left(\phi-\phi_{P}\right)+\frac{\rho^{2}}{R^{2}}\right]^{1 / 2} \simeq R-\rho \sin \theta_{P} \cos \left(\phi-\phi_{P}\right)+\frac{1}{2} \frac{\rho^{2}}{R}
$$

The integration for the fields contains the extra phase factor $\exp \left[i k \rho^{2} /(2 R)\right]$ when compared to the fields calculated in the far field approximation. 
Define the dimensionless variables

$$
p=\frac{\rho}{a_{\text {in }}}, \quad r=\frac{R}{a_{\text {in }}}, \quad \bar{a}=\frac{a}{a_{\text {in }}}, \quad g=\frac{a_{\text {out }}}{a_{\text {in }}}, \quad \eta=\frac{\gamma u}{2 \beta r}, \Rightarrow \frac{k \rho^{2}}{2 R}=\eta p^{2}
$$

Define the complex function

$$
\begin{aligned}
S[\bar{a} ; \bar{k}, r] & =\frac{1}{a_{i n}^{2}} \int_{0}^{a} \rho J_{1}(\bar{k} \rho) K_{1}(\alpha a) \exp \left[i \frac{k \rho^{2}}{2 R}\right] d \rho \\
& =\int_{0}^{\bar{a}} p J_{1}\left(\frac{1}{\beta} u t p\right) K_{1}\left(\frac{1}{\beta} u p\right) \exp \left[i \eta p^{2}\right] d p
\end{aligned}
$$

Then following similar steps as in the previous section, it follows that the Fourier transforms of the transverse electric fields are

$$
\left[\begin{array}{c}
E_{\omega, x} \\
E_{\omega, y}
\end{array}\right]=-\frac{k q \alpha}{\pi v} \frac{e^{i k R}}{R}[S(g ; \bar{k}, r)-S(1 ; \bar{k}, r)]\left[\begin{array}{c}
\cos \phi_{P} \\
\sin \phi_{P}
\end{array}\right]
$$

The angular spectral distribution is

$$
\frac{d^{2} W}{d \Omega d \omega}=\frac{1}{2} \frac{q^{2}}{\pi^{2} \beta c}\left(\gamma u^{2}\right)^{2}|[S(g ; \bar{k})-S(1 ; \bar{k})]|^{2}
$$

The frequency spectrum is found by integrating over the solid angle

$$
\frac{d W}{d \omega}=\frac{4 \pi}{\gamma^{2}} \int_{0}^{\gamma} \frac{d^{2} W}{d \Omega d \omega} \frac{t}{\sqrt{1-t^{2} / \gamma^{2}}} d t
$$

Define the function

$$
D(g, u, r)=\int_{0}^{\gamma} d t \frac{t}{\sqrt{1-t^{2} / \gamma^{2}}}|[S(g ; \bar{k}, r)-S(1 ; \bar{k}, r)]|^{2}
$$

The number of photons emitted by a single charged particle into a bandwidth $\Delta \omega$ is therefore

$$
\Delta N_{p h}=\left(\frac{1}{\pi \beta} \alpha_{f}\right) u^{4} D(g, u, r) \frac{\Delta \omega}{\omega}
$$

This depends on the inner radius $a_{\text {in }}$ through the scaled variables $g=a_{\text {out }} / a_{\text {in }}, r=R / a_{\text {in }}, u=$ $\omega / \omega_{c}$.

\subsubsection{Particle offset from the center of target}

Our final aim is to find the spectral distribution from a bunch of particles. Towards that end we first need to know the field distribution from a particle offset from the center of the target. The target is in the $z=0$ plane and the particle moves with uniform velocity $v$ along the $\mathrm{z}$ axis. Figure 4 shows a sketch of the target with center at $\mathrm{O}$, the particle is at $\mathrm{B}$ and the field on the 

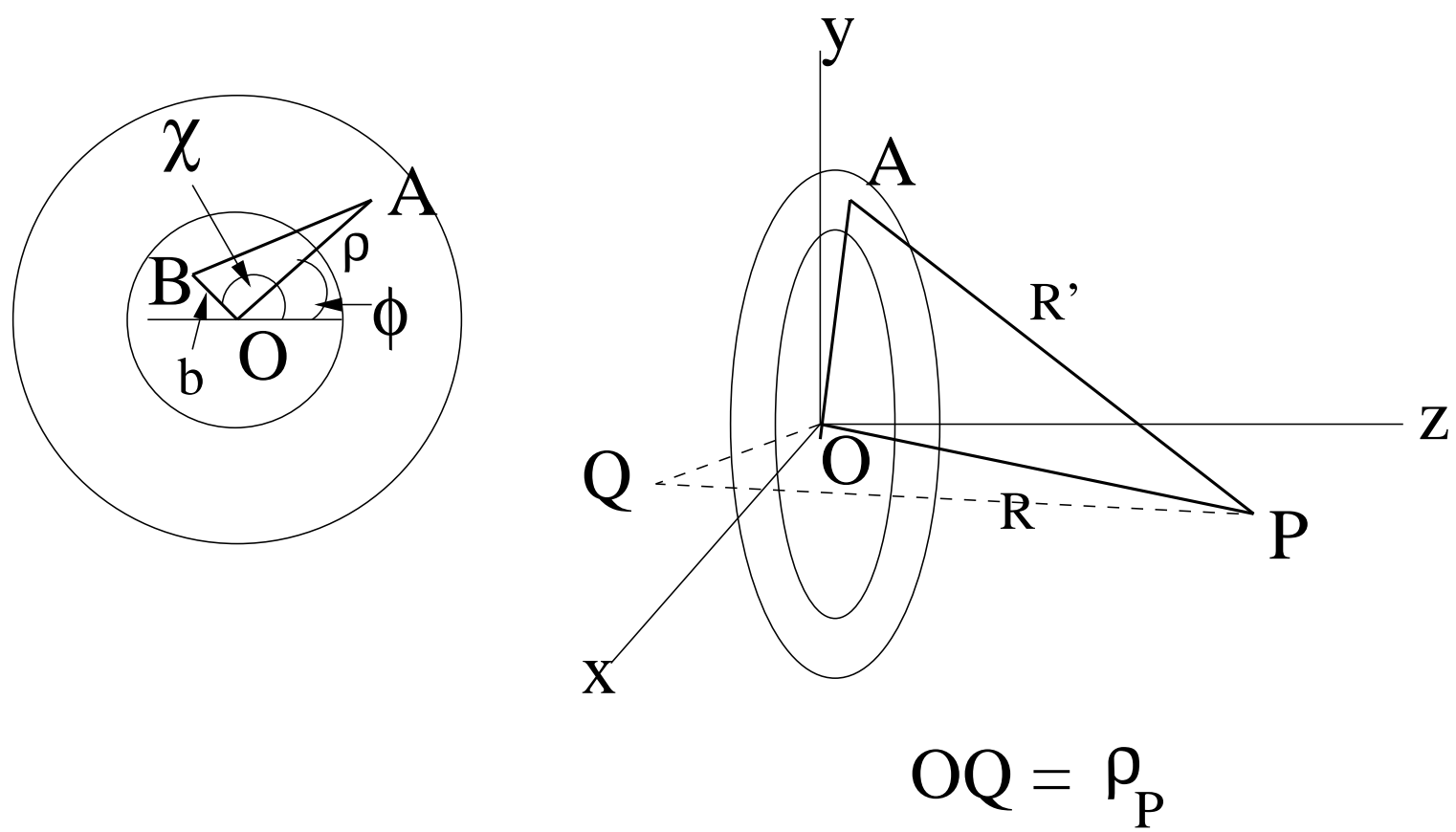

Figure 4: The target centered at $\mathrm{O}$ is an annulus of inner radius $a_{\text {in }}$ and outer radius $a_{\text {out }}$. $\mathrm{B}$ is the location of the particle offset by a distance $b$ from the center of the target, $\mathrm{A}$ is the arbitrary location on the target for the field calculation. In the figure on the right, $\mathrm{P}$ is the point of observation, $\mathrm{Q}$ is the projection of $\mathrm{P}$ on to $x-y$ plane.

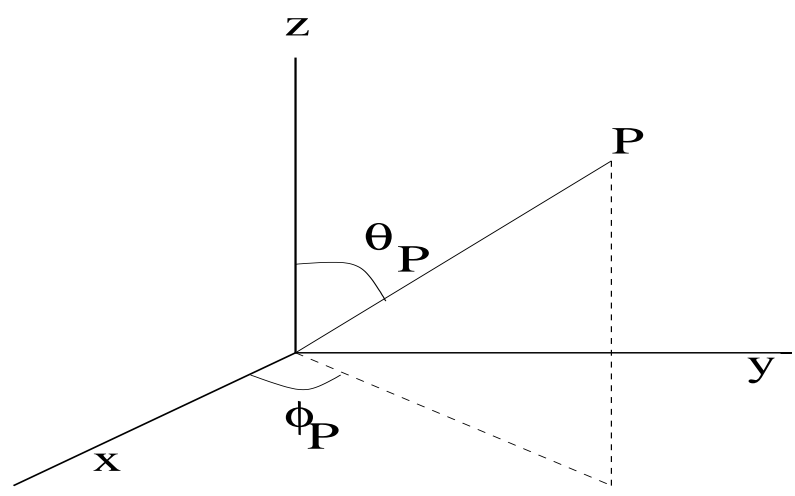

Figure 5: $\mathrm{P}$ is the observation point, angle $\theta_{P}$ is the angle with the $\mathrm{z}$ axis and $\phi_{P}$ is the angle with the $\mathrm{x}$ axis made by the projection onto the $\mathrm{x}-\mathrm{y}$ plane. 
target is calculated at point A. Again we use polar coordinates: particle at B has coordinates $(b, \chi)$ while point A has coordinates $(\rho, \phi)$ with respect to the center O. The distance of A relative to the particle at $B$ is

$$
r_{\perp}=\left[\rho^{2}+b^{2}-2 \rho b \cos (\chi-\phi)\right]^{1 / 2}
$$

while the separation along the $(x, y)$ axes are individually

$$
x=x_{A}-x_{B}=\rho \cos \phi-b \cos \chi, \quad y=y_{A}-y_{B}=\rho \sin \phi-b \sin \chi
$$

Hence the Fourier transforms of the transverse fields at A are

$$
\left[\begin{array}{c}
E_{\omega, x} \\
E_{\omega, y}
\end{array}\right]=\frac{q \alpha}{\pi v} \frac{K_{1}\left(\alpha \rho_{\perp}\right)}{\rho_{\perp}}\left[\begin{array}{c}
\rho \cos \phi-b \cos \chi \\
\rho \sin \phi-b \sin \chi
\end{array}\right]
$$

As before $R^{\prime}$ is the distance of the observation point $\mathrm{P}$ from the location of the field, thus

$$
R^{\prime}=\left[\left(x_{P}-\rho \cos \phi\right)^{2}+\left(y_{P}-\rho \sin \phi\right)^{2}+z_{P}^{2}\right]^{1 / 2}
$$

where $\left(x_{P}, y_{P}, z_{P}\right)=\left(\rho_{P} \cos \phi_{P}, \rho_{P} \sin \phi_{P}, z_{P}\right)$ are the coordinates of the point P. Figure 5 shows the relevant angles $\theta_{P}, \phi_{P}$.

Integrating over the annulus, the fields from the entire target at the point $\mathrm{P}$ are (using scalar diffraction theory)

$$
\left[\begin{array}{c}
E_{\omega, x} \\
E_{\omega, y}
\end{array}\right]=-\frac{i k}{2 \pi} \frac{q \alpha}{\pi v} \int_{a_{\text {in }}}^{a_{\text {out }}} \int_{0}^{2 \pi} \rho d \rho d \phi \frac{e^{i k R^{\prime}}}{R^{\prime}} \frac{K_{1}\left(\alpha \rho_{\perp}\right)}{\rho_{\perp}}\left[\begin{array}{c}
\rho \cos \phi-b \cos \chi \\
\rho \sin \phi-b \sin \chi
\end{array}\right]
$$

Far field spectrum

We assume that the point $\mathrm{P}$ is sufficiently far from the target that the far field approximation is valid. The phase term is expanded as

$$
\frac{e^{i k R^{\prime}}}{R^{\prime}}=\frac{e^{i k R}}{R} e^{-i \bar{k} \rho \cos \left(\phi-\phi_{P}\right)}
$$

where $\bar{k}=k \sin \theta_{P}, \theta_{P}$ is the angle made by OP with the $z$ axis or $\sin \theta_{P}=\rho_{P} / R$.

The integrations are simplified if we write the integrands as derivatives with respect to variables that are not integrated. We note first that

$$
\left[\begin{array}{c}
\frac{\partial}{\partial b} \\
\frac{\partial}{\partial \chi}
\end{array}\right] K_{0}\left(\alpha \rho_{\perp}\right)=\frac{\alpha K_{1}\left(\alpha \rho_{\perp}\right)}{\rho_{\perp}}\left[\begin{array}{c}
\rho \cos (\phi-\chi)-b \\
b \rho \sin (\phi-\chi)
\end{array}\right]
$$

Next we transform the fields to a frame rotated by angle $\chi$ with respect to the $(x, y)$ axes, i.e.

$$
\left[\begin{array}{l}
E_{1} \\
E_{2}
\end{array}\right]=\left[\begin{array}{cc}
\cos \chi & \sin \chi \\
-\sin \chi & \cos \chi
\end{array}\right]\left[\begin{array}{c}
E_{\omega, x} \\
E_{\omega, y}
\end{array}\right]
$$


Then using the derivative expressions above, we find

$$
\left[\begin{array}{c}
E_{1} \\
E_{2}
\end{array}\right]=-\frac{i k}{2 \pi} \frac{q}{\pi v} \frac{e^{i k R}}{R}\left[\begin{array}{c}
\frac{\partial}{\partial b} \\
\frac{1}{b} \frac{\partial}{\partial \chi}
\end{array}\right] \int_{a_{\text {in }}}^{a_{\text {out }}} \int_{0}^{2 \pi} d \rho d \phi \rho K_{0}\left(\alpha \rho_{\perp}\right) e^{-i \bar{k} \rho \cos \left(\phi-\phi_{P}\right)}
$$

The double integral can be factorized into the product of single integrals by using the expansions

$$
\begin{aligned}
K_{0}\left(\alpha\left[\rho^{2}+b^{2}-2 b \rho \cos (\phi-\chi)\right]^{1 / 2}\right) & =\sum_{n=-\infty}^{\infty} I_{n}(\alpha b) K_{n}(\alpha \rho) e^{i n(\phi-\chi)} \\
\exp \left[-i \bar{k} \rho \cos \left(\phi-\phi_{P}\right)\right] & =\sum_{n=-\infty}^{\infty}(-i)^{n} J_{n}(\bar{k} \rho) e^{i n\left(\phi-\phi_{P}\right)}
\end{aligned}
$$

The integration over $\phi$ is trivial and the double integral reduces to

$$
\begin{aligned}
H \equiv \int_{a_{\text {in }}}^{a_{\text {out }}} \int_{0}^{2 \pi} d \rho d \phi \rho K_{0}\left(\alpha \rho_{\perp}\right) e^{-i \bar{k} \rho \cos \left(\phi-\phi_{P}\right)}= & 2 \pi \sum_{n=-\infty}^{\infty}(-i)^{n} I_{n}(\alpha b) e^{-i n\left(\chi-\phi_{P}\right)} \\
& \times \int_{a_{\text {in }}}^{a_{\text {out }}} \rho J_{n}(\bar{k} \rho) K_{n}(\alpha \rho) d \rho
\end{aligned}
$$

The integration over $\rho$ can be done symbolically using Mathematica [7] which yields

$$
\begin{aligned}
\int_{0}^{a} \rho J_{n}(\bar{k} \rho) K_{n}(\alpha \rho) d \rho & =\frac{a}{\bar{k}^{2}+\alpha^{2}}\left[\bar{k} J_{n+1}(\bar{k} a) K_{n}(\alpha a)-\alpha J_{n}(\bar{k} a) K_{n+1}(\alpha a)\right] \\
& =-\frac{a}{\bar{k}^{2}+\alpha^{2}}\left[\bar{k} J_{n-1}(\bar{k} a) K_{n}(\alpha a)+\alpha J_{n}(\bar{k} a) K_{n-1}(\alpha a)\right]
\end{aligned}
$$

where recurrence relations for the Bessel functions were used. Then defining a function $T_{n}(a ; \bar{k})$ similar to the one defined in Equation (12) in Section 3.1.1,

$$
T_{n}(a ; k)=-a\left[\bar{k} J_{n-1}(\bar{k} a) K_{n}(\alpha a)+\alpha J_{n}(\bar{k} a) K_{n-1}(\alpha a)\right]
$$

After some further simplifications we can write

$$
H=\frac{2 \pi}{\bar{k}^{2}+\alpha^{2}} \sum_{n=0}^{\infty} C_{n} I_{n}(\alpha b)\left[T_{n}\left(a_{\text {out }} ; \bar{k}\right)-T_{n}\left(a_{i n} ; \bar{k}\right)\right] \cos n\left(\chi-\phi_{P}\right)
$$

where

$$
C_{n}=1 \text { for } n=0 ; \quad C_{n}=2(-i)^{n} \text { for } n \geq 1
$$

and the rotated fields are

$$
\left[\begin{array}{c}
E_{1} \\
E_{2}
\end{array}\right]=-\frac{i k \alpha q}{\pi v\left(\bar{k}^{2}+\alpha^{2}\right)} \frac{e^{i k R}}{R} \sum_{n=0} C_{n}\left[T_{n}\left(a_{\text {out }} ; \bar{k}\right)-T_{n}\left(a_{i n} ; \bar{k}\right)\right]\left[\begin{array}{c}
I_{n}^{\prime}(\alpha b) \cos \left[n\left(\chi-\phi_{P}\right)\right] \\
-n \frac{I_{n}(\alpha b)}{\alpha b} \sin \left[n\left(\chi-\phi_{P}\right)\right]
\end{array}\right]
$$

The fields in the lab frame $\left(E_{\omega, x}, E_{\omega, y}\right)$ are obtained by applying the inverse rotation

$$
\left[\begin{array}{c}
E_{\omega, x} \\
E_{\omega, y}
\end{array}\right]=\left[\begin{array}{cc}
\cos \chi & -\sin \chi \\
\sin \chi & \cos \chi
\end{array}\right]\left[\begin{array}{c}
E_{1} \\
E_{2}
\end{array}\right]
$$




\section{$\underline{\text { Limit of zero offset }}$}

Before proceeding further, we first check that the expressions derived here reduce to the expressions derived in the previous sub-section in the limit that the offset is zero.

First we note that

$$
\lim _{\chi \rightarrow 0}\left[\begin{array}{c}
E_{\omega, x} \\
E_{\omega, y}
\end{array}\right]=\left[\begin{array}{l}
E_{1} \\
E_{2}
\end{array}\right]
$$

Now using the fact that

$$
\lim _{b \rightarrow 0} \frac{I_{n}(\alpha b)}{b}=\frac{\alpha}{2} \delta_{n, 1}
$$

Then only the $n=1$ term in the sum contributes and we have

$$
\lim _{b, \chi \rightarrow 0}\left[\begin{array}{c}
E_{\omega, x} \\
E_{\omega, y}
\end{array}\right]=-\frac{k q \alpha}{\pi v\left(\bar{k}^{2}+\alpha^{2}\right)} \frac{e^{i k R}}{R}\left[T_{1}\left(a_{\text {out }} ; \bar{k}\right)-T_{1}\left(a_{i n} ; \bar{k}\right)\right]\left[\begin{array}{c}
\cos \phi_{P} \\
\sin \phi_{P}
\end{array}\right]
$$

These expressions agree with the expressions in Equation (13) derived earlier.

We return now to the case with offset. The differential angular spectrum is proportional to the square of the absolute norm which is preserved under rotations,

$$
\left|E_{\omega, x}\right|^{2}+\left|E_{\omega, y}\right|^{2}=\left|E_{1}\right|^{2}+\left|E_{2}\right|^{2}
$$

Hence the differential angular spectrum is given by

$$
\frac{d^{2} W}{d \Omega d \omega}=\frac{1}{2} \beta c R^{2}\left[\left|E_{1}\right|^{2}+\left|E_{2}\right|^{2}\right]
$$

The spectrum from a single particle therefore is

$$
\left.\frac{d^{2} W}{d \Omega d \omega}\right|_{\text {particle }}=\frac{1}{2} \beta c\left[\frac{k q \alpha}{\pi v\left(\bar{k}^{2}+\alpha^{2}\right)}\right]^{2} \sum_{m=0} \sum_{n=0} C_{m} C_{n}^{*} V_{m n}
$$

where we have defined

$$
\begin{aligned}
V_{m n}= & U_{m n}\left\{I_{m}^{\prime}(\alpha b) I_{n}^{\prime}(\alpha b) \cos m\left(\chi-\phi_{P}\right) \cos n\left(\chi-\phi_{P}\right)\right. \\
& \left.+m n \frac{I_{m}(\alpha b) I_{n}(\alpha b)}{\alpha^{2} b^{2}} \sin m\left(\chi-\phi_{P}\right) \sin n\left(\chi-\phi_{P}\right)\right\} \\
U_{m n}= & {\left[T_{m}\left(a_{\text {out }} ; \bar{k}\right)-T_{m}\left(a_{\text {in }} ; \bar{k}\right)\right]\left[T_{n}\left(a_{\text {out }} ; \bar{k}\right)-T_{n}\left(a_{\text {in }} ; \bar{k}\right)\right] }
\end{aligned}
$$

It is helpful to use the scaled variables $g, u, t$ introduced in Equation (19). Then

$$
k=\frac{\gamma u}{\beta a_{\text {in }}}, \bar{k}=k \sin \theta_{P}=\frac{t u}{\beta a_{\text {in }}}, \quad \alpha=\frac{u}{\beta a_{\text {in }}}, \quad\left(\frac{k q \alpha}{\pi v\left(\bar{k}^{2}+\alpha^{2}\right)}\right)^{2}=\left(\frac{q \gamma}{\pi v}\right)^{2} \frac{1}{\left(1+t^{2}\right)^{2}}
$$

Furthermore

$$
\begin{aligned}
{\left[T_{m}\left(a_{\text {out }} ; \bar{k}\right)-T_{m}\left(a_{i n} ; \bar{k}\right)\right]=} & -\frac{u}{\beta}\left\{g\left[t J_{m-1}\left(\frac{g u t}{\beta}\right) K_{m}\left(\frac{g u}{\beta}\right)+J_{m}\left(\frac{g u t}{\beta}\right) K_{m-1}\left(\frac{g u}{\beta}\right)\right]\right. \\
& \left.-\left[t J_{m-1}\left(\frac{u t}{\beta}\right) K_{m}\left(\frac{u}{\beta}\right)+J_{m}\left(\frac{u t}{\beta}\right) K_{m-1}\left(\frac{u}{\beta}\right)\right]\right\}
\end{aligned}
$$


Hence $U_{m n}(g, u, t)$ is only a function of the scaled variables $g, u, t$ but does not depend on the inner radius $a_{i n}$. Similarly we define the scaled offset

$$
b_{s}=\frac{b}{a_{i n}}, \Rightarrow \alpha b=b_{s} \frac{u}{\beta}
$$

This shows that $V_{m n}\left(g, u, t, b_{s}\right)$ is also independent of the inner aperture $a_{i n}$.

Since $V_{m n}$ is real and symmetric under the interchange of indices, we can write

$$
\sum_{m=0} \sum_{n=0} C_{m} C_{n}^{*} V_{m n}=\frac{1}{2} \sum_{m=0} \sum_{n=0}\left(C_{m} C_{n}^{*}+C_{m}^{*} C_{n}\right) V_{m n}
$$

Using the definition of $C_{m}$ we obtain

$$
\begin{aligned}
C_{m}+C_{m}^{*} & =0 \quad m \text { odd } \\
& =4(-1)^{m / 2} \quad m \text { even } \\
C_{m} C_{n}^{*}+C_{m}^{*} C_{n} & =0 \quad m-n \text { odd } \\
& =8(-1)^{(m-n) / 2} \quad m-n \text { even }
\end{aligned}
$$

Hence

$$
\begin{aligned}
\left.\frac{d^{2} W}{d \Omega d \omega}\right|_{\text {particle }}(g, u, t, b, \chi)= & \frac{1}{2} \beta c\left(\frac{q \gamma}{\pi v}\right)^{2} \frac{1}{\left(1+t^{2}\right)^{2}}\left[V_{00}+4 \sum_{m=2,4, \ldots}(-1)^{m / 2} V_{m 0}\right. \\
& \left.+4 \sum_{m=1} \sum_{n=1 ;|m-n|=\text { even }}(-1)^{(m-n) / 2} V_{m n}\right]
\end{aligned}
$$

In the limit that the offset goes to zero, this reduces to

$$
\left.\lim _{b \rightarrow 0} \frac{d^{2} W}{d \Omega d \omega}\right|_{\text {particle }}=\frac{1}{2} \beta c\left(\frac{q \gamma}{\pi v}\right)^{2} \frac{1}{\left(1+t^{2}\right)^{2}} U_{11}
$$

which agrees with the expression in Equation (20).

The dependence of the single particle spectrum on the parameters $(g, u, t)$ is similar to that seen in Figures 1 and 2. Figure 6 shows the dependence of the single particle spectrum on the angle of observation for two different offsets $b$ from the center of the hole. With an increased offset the particle is closer to the material of the target resulting in a larger radiation flux .

The intensities of the different polarizations can be found from the components of the electric field. From Equation 42 it follows that

$$
\begin{aligned}
E_{\omega, x}= & -\frac{i k \alpha q}{\pi v\left(\bar{k}^{2}+\alpha^{2}\right)} \frac{e^{i k R}}{R} \sum_{n} C_{n}\left[T_{n}\left(a_{\text {out }} ; k\right)-T_{n}\left(a_{\text {in }} ; k\right)\right] \\
& \times\left\{I_{n}^{\prime}(\alpha b) \cos \left[n\left(\chi-\phi_{P}\right)\right] \cos \chi+n \frac{I_{n}(\alpha b)}{\alpha b} \sin \left[n\left(\chi-\phi_{P}\right)\right] \sin \chi\right\} \\
E_{\omega, y}= & -\frac{i k \alpha q}{\pi v\left(\bar{k}^{2}+\alpha^{2}\right)} \frac{e^{i k R}}{R} \sum_{n} C_{n}\left[T_{n}\left(a_{\text {out }} ; k\right)-T_{n}\left(a_{i n} ; k\right)\right] \\
& \times\left\{I_{n}^{\prime}(\alpha b) \cos \left[n\left(\chi-\phi_{P}\right)\right] \sin \chi-n \frac{I_{n}(\alpha b)}{\alpha b} \sin \left[n\left(\chi-\phi_{P}\right)\right] \cos \chi\right\}
\end{aligned}
$$




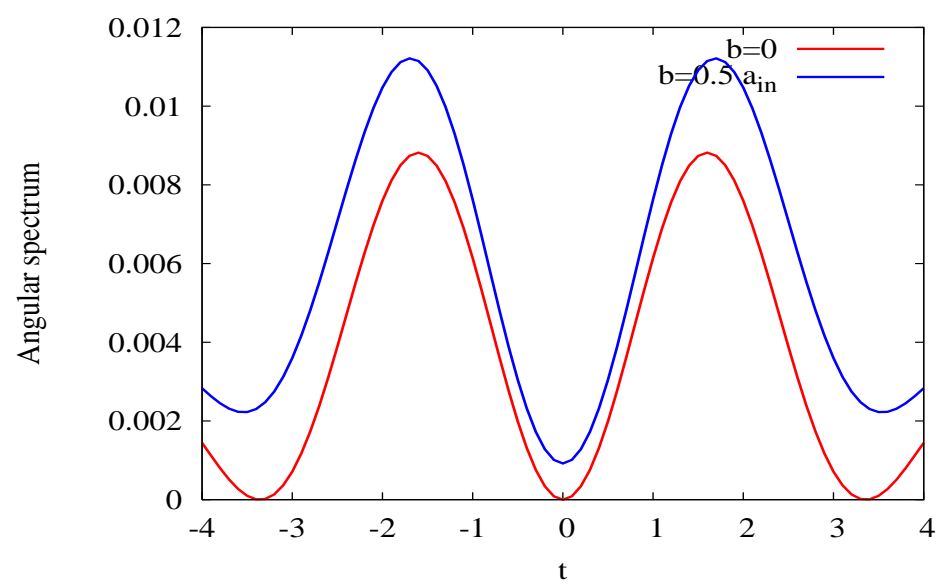

Figure 6: The differential angular spectrum from a single particle vs the angle of observation $\left(t=\gamma \sin \theta_{P}\right)$ with two different offsets $b=0$ and $b=0.5 a_{i n}$. Other parameter values are fixed at $g=1.3, u=1, \chi=0, \phi_{P}=\pi / 4$. With non-zero offset, both the minimum and the maximum value of the flux increases. Note that the position of th maximum also changes with the offset.

Hence it follows that the intensities of the two polarizations are given by

$$
\begin{aligned}
\left.\frac{d^{2} W^{x, y}}{d \Omega d \omega}\right|_{\text {particle }}(g, u, t, b, \chi)= & \frac{1}{2} \beta c\left(\frac{q \gamma}{\pi v}\right)^{2} \frac{1}{\left(1+t^{2}\right)^{2}}\left[V_{00}^{x, y}+4 \sum_{m=2,4, \ldots}(-1)^{m / 2} V_{m 0}^{x, y}\right. \\
& \left.+4 \sum_{m=1} \sum_{n=1 ;|m-n|=\text { even }}(-1)^{(m-n) / 2} V_{m n}^{x, y}\right]
\end{aligned}
$$

where

$$
\begin{aligned}
V_{m n}^{x}= & U_{m n}\left[I_{m}^{\prime}(\alpha b) \cos \left[m\left(\chi-\phi_{P}\right)\right] \cos \chi+m \frac{I_{m}(\alpha b)}{\alpha b} \sin \left[m\left(\chi-\phi_{P}\right)\right] \sin \chi\right] \\
& \times\left[I_{n}^{\prime}(\alpha b) \cos \left[n\left(\chi-\phi_{P}\right)\right] \cos \chi+n \frac{I_{n}(\alpha b)}{\alpha b} \sin \left[n\left(\chi-\phi_{P}\right)\right] \sin \chi\right] \\
V_{m n}^{y}= & U_{m n}\left[I_{m}^{\prime}(\alpha b) \cos \left[m\left(\chi-\phi_{P}\right)\right] \sin \chi-m \frac{I_{m}(\alpha b)}{\alpha b} \sin \left[m\left(\chi-\phi_{P}\right)\right] \cos \chi\right] \\
& \times\left[I_{n}^{\prime}(\alpha b) \cos \left[n\left(\chi-\phi_{P}\right)\right] \sin \chi-n \frac{I_{n}(\alpha b)}{\alpha b} \sin \left[n\left(\chi-\phi_{P}\right)\right] \cos \chi\right.
\end{aligned}
$$

We will use the polarized intensities to examine their sensitivity to beam parameters in the next section.

\subsection{Spectrum from a bunch}

So far we've dealt with the spectrum from a single particle traveling through the hole. Now we'll consider the spectrum from a typical bunch. We assume here a Gaussian distribution of $\mathrm{N}$ 
particles with transverse rms sizes $\sigma_{x}, \sigma_{y}$. We also assume that the bunch center is offset from the center of the target with offsets $\left(x_{0}, y_{0}\right)$. Then

$$
\begin{aligned}
\rho(x, y) & =\frac{N}{2 \pi \sigma_{x} \sigma_{y}} \exp \left[-\frac{\left(x-x_{0}\right)^{2}}{2 \sigma_{x}^{2}}-\frac{\left(y-y_{0}\right)^{2}}{2 \sigma_{y}^{2}}\right] \\
& =\frac{N}{2 \pi \sigma_{x} \sigma_{y}} \exp \left[-\frac{\left(b_{s} \cos \chi-x_{0, s}\right)^{2}}{2 \sigma_{x, s}^{2}}-\frac{\left(b_{s} \sin \chi-y_{0, s}\right)^{2}}{2 \sigma_{y, s}^{2}}\right] \\
& \equiv \frac{N}{2 \pi \sigma_{x} \sigma_{y}} \rho_{s}\left(b_{s}, \chi, x_{0, s}, y_{0, s}, \sigma_{x, s}, \sigma_{y, s}\right)
\end{aligned}
$$

The last equality defines the scaled density $\rho_{s}$ and we have scaled the other variables by $a_{i n}$, the inner radius of the hole,

$$
b_{s}=\frac{b}{a_{i n}}, x_{0, s}=\frac{x_{0}}{a_{i n}}, y_{0, s}=\frac{y_{0}}{a_{i n}}, \sigma_{x, s}=\frac{\sigma_{x}}{a_{i n}}, \sigma_{y, s}=\frac{\sigma_{y}}{a_{i n}}
$$

The differential angular spectrum averaged over the bunch distribution is

$$
\begin{aligned}
\left.\frac{d^{2} W}{d \Omega d \omega}\right|_{\text {bunch }} & =\left.\int b d b \int d \chi \rho(b, \chi) \frac{d^{2} W}{d \Omega d \omega}\right|_{\text {particle }}=\left.a_{\text {in }}^{2} \int b_{s} d b_{s} \int d \chi \rho\left(b_{s}, \chi\right) \frac{d^{2} W}{d \Omega d \omega}\right|_{\text {particle }} \\
& =\left.\frac{N}{2 \pi \sigma_{x, s} \sigma_{y, s}} \int b_{s} d b_{s} \int d \chi \rho_{s} \frac{d^{2} W}{d \Omega d \omega}\right|_{\text {particle }}
\end{aligned}
$$

It is important to note that the spectrum depends only on the scaled variables introduced in Equation (60) but not on the absolute values of $a_{i n},\left(\sigma_{x}, \sigma_{y}\right),\left(x_{0}, y_{0}\right)$. Hence this is a universal expression; the only dependence on machine specific parameters is on the beam energy and the bunch intensity.

The two-dimensional integrals over $\left(b_{s}, \chi\right)$ can be factored as the product of single integrals over $b_{s}$ and $\chi$ individually. However the integration over $\chi$ introduces a triple summation and the integrals over $b$ cannot be performed analytically. Instead we will evaluate the integrations over the bunch numerically.

The bunch spectrum has the same dependence on the target size ratio $g$, the scaled frequency $u$ and the scaled angle of observation $t=\gamma \sin \theta_{P}$ as the single particle spectrum. Figure 7 and 8 show the dependence of the spectrum on $(g, t)$ and $(u, t)$ respectively. As before, the intensity initially increases with $g$ but flattens for $g>2.5$. In the sequel I will set $g=1.3$ in order to limit the size of the target. Larger sizes than this if feasible result in significantly larger ODR intensities mostly at low frequencies but do not change the intensity much at high frequencies. Figure 8 shows that the angular spectrum as a function of frequency peaks in the vicinity of $u=1$ or $\omega=\omega_{c}$ when viewed at the angle of maximum intensity corresponding to $t=1.6$. At other angles, the first peak moves to other values of $u$ and the peaks are of comparable height.

\section{Sensitivity to beam parameters}

The beam parameters that we wish to measure with ODR are the beam sizes and the beam positions. The angular spectral distribution is sensitive to these parameters and we examine 


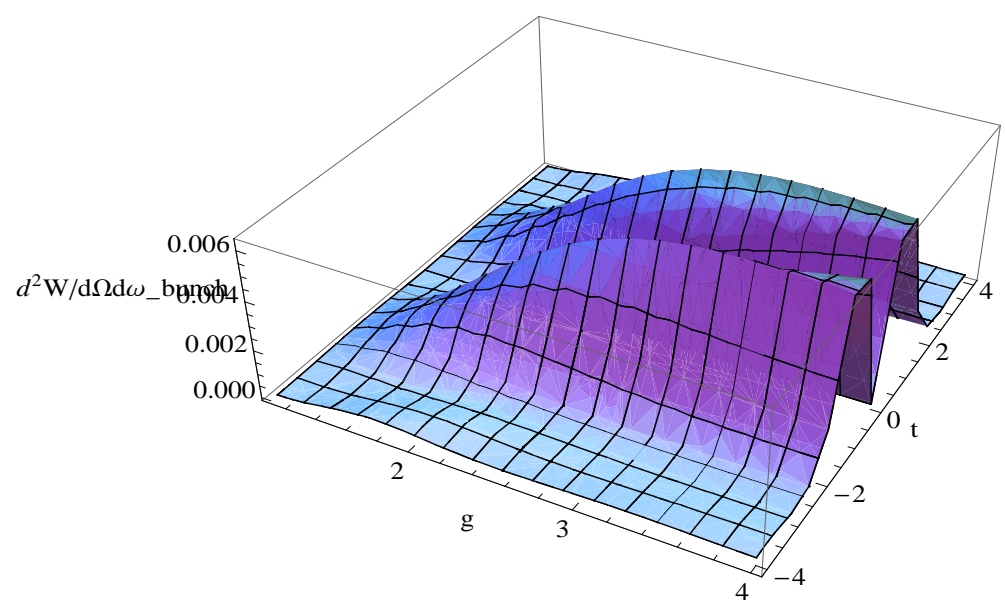

Figure 7: The differential angular spectrum from a bunch as a function of $g=a_{\text {out }} / a_{\text {in }}$ and the angular variable $t=\gamma \sin \theta_{P}$ at constant $u=1$. As a function of $g$, the spectrum is relatively flat for $g>2.5$.
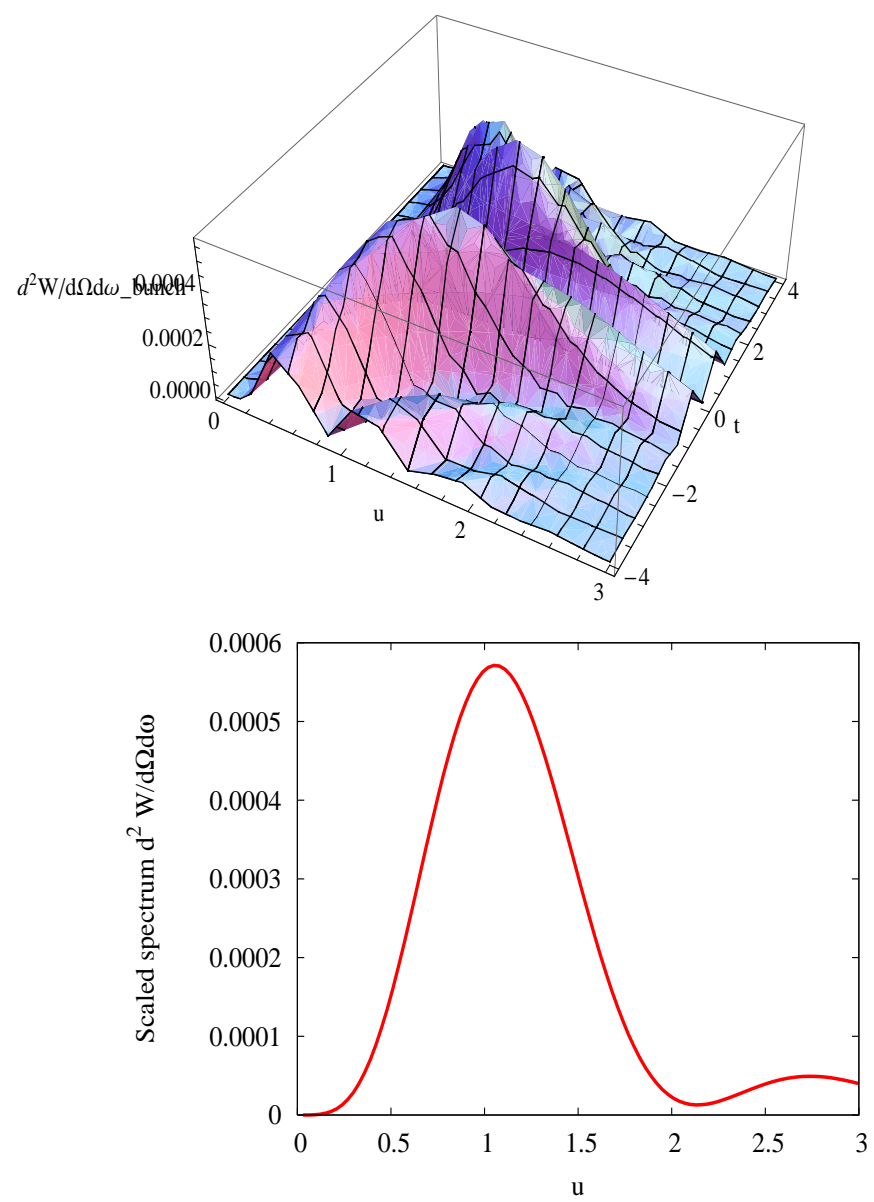

Figure 8: Top: The differential angular spectrum from a bunch as a function of the scaled frequency $u$ and the angular variable $t=\gamma \sin \theta_{P}$ at constant $g=1.3$. Bottom: The spectrum as a function of $u$ at $t=1.6$, and other parameters at the same values. The spectrum peaks at around $u=1.1$. 


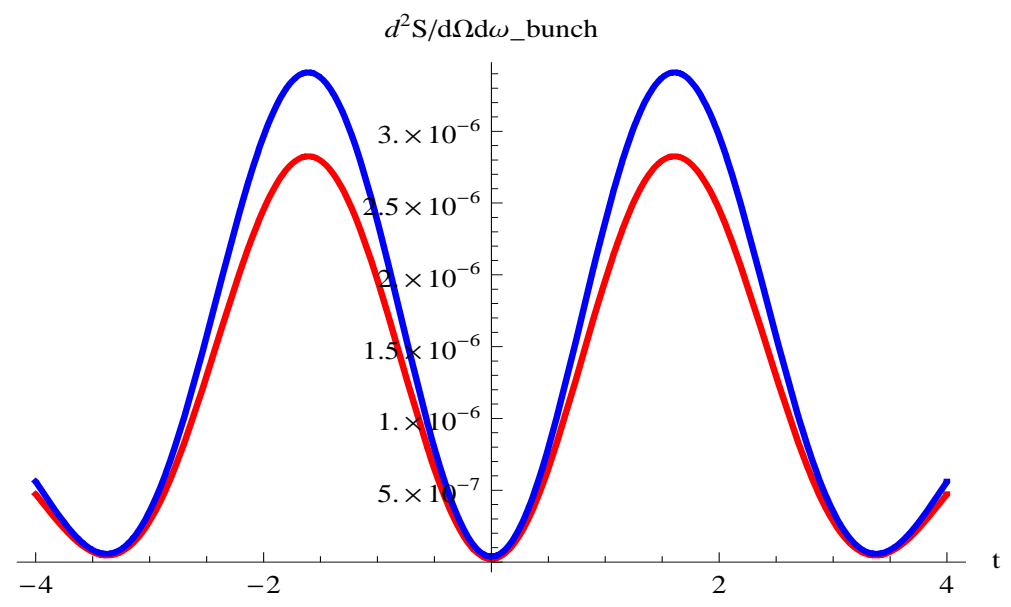

Figure 9: The differential angular spectrum of the horizontal polarization $d^{2} S^{x} / d \Omega d \omega$ for two different beam sizes. The red curve is obtained for the scaled beam size $\sigma_{x, s}=0.1$ while the blue curve is obtained for $\sigma_{x, s}=0.12$. Both the minimum and maximum values change with the beam size, the minimum increases more rapidly on a relative scale.

here the dependence on these parameters. Since the detector will have a finite bandwidth in frequency acceptance, we include this in our analysis. We define a finite bandwidth spectrum by integrating over the frequency as

$$
\frac{d^{2} S}{d \Omega d \omega}=\int_{\Delta u} d u \frac{d^{2} W}{d \Omega d \omega}
$$

Here we assume a $1 \%$ bandwidth for $\Delta u$.

Consider the sensitivity of the separate polarizations to changes in the beam size. Figure 9 shows the horizontal polarization intensity $d^{2} S^{x} / d \Omega d \omega$ as a function of the angle variable $t$ for two different values of the scaled beam size $\sigma_{x, s}$. When the beam size is larger filling more of the aperture, the intensity increases at all observation angles. While the absolute change in the maximum intensity is larger with larger beam size, the relative change in the minimum intensity (at $t=0$ ) is larger than that in the maximum intensity.

This was exploited in the KEK experiments [1] where the beam size was determined from the ratio of the minimum to the maximum of the angular spectrum and we apply the same technique here. The top plot in Figure 10 shows the ratio of the minimum to maximum of the horizontal and vertical polarization intensities, $d^{2} S^{x} / d \Omega d \omega$ and $d^{2} S^{y} / d \Omega d \omega$ respectively, as a function of the scaled horizontal beam size $\sigma_{x, s}$. We mention an important point here: the ratio is independent of the energy, bunch intensity etc but depends only on the scaled parameters $(g, u, t)$ and scaled beam parameters $\left(\sigma_{x, s}, \sigma_{y, s}, x_{0, s}, y_{0, s}\right)$. Hence as long as the scaled variables have the same values, these ratios will be the same for the Tevatron, LHC etc. The figure shows the data points calculated from the expressions in Equations (55), (61) and (62) as well as a quadratic fit through these points. The ratio for the horizontal polarization increases quadratically with the horizontal beam size but the vertical polarization is insensitive to the change in horizontal beam size. Since the fit to the horizontal spectrum is quadratic, a $1 \%$ increase in the 
horizontal beam size results in a $2 \%$ increase in the minimum to maximum ratio. This suggests that if intensity differences at the level of $2 \%$ can be resolved, then beam size changes at the level of $1 \%$ can be detected. The bottom plot in Figure 10 shows the ratio for the horizontal polarization at three frequencies: $u=0.5,1$. The ratio increases quadratically with $\sigma_{x}$ at all frequencies. Furthermore, the ratio increases with $u$ showing that the sensitivity of the beam size measurement increases with frequency.

We consider now the sensitivity of the angular spectrum to changes in the beam offsets. To be useful for diagnostics, the spectrum should be sensitive to changes in offset which are fractions of a beam size. We find that when the offsets are in the range $(0-1) \times \sigma$, the maximum of the angular spectrum does not change significantly. However the minimum of the spectrum at $t=0$ does change rapidly. So we examine instead the relative change in the minimum intensity as the offset changes. Figure 11 shows the relative change of $d^{2} S^{x}(t=0) / d \Omega d \omega$ to changes in the beam offset shown in units of the beam size for three values of the frequency $u$. We observe that as the horizontal offset increases from zero to one times the beam size, the minimum horizontal polarization doubles in value. The relative change in the minimum is nearly the same for the three values of $u$, so at these frequencies the relative change in the minimum is not very sensitive to frequency. The bottom plot in Figure 11 shows the ratio of the minimum to the maximum of the horizontal polarization. This ratio also increases quadratically with the offset and more importantly is sensitive to the frequency, increasing at higher frequencies. These results show that if changes in the minimum intensity at the level of a few $\%$ can be measured, then changes in beam offsets of fractions of a beam size can be detected.

\section{Photon yield}

We start by calculating the differential spectrum for a single particle that is offset from the center of the hole. This will generalize the results in Section 3.1.1. We will then use this result to calculate the photon yield from a bunch and its dependence on beam and target parameters.

The differential spectrum for a single particle is found by integrating the single particle differential angular spectrum over the solid angle,

$$
\left.\frac{d W}{d \omega}\right|_{p a r t}=\int \frac{d^{2} W}{d \Omega d \omega}{ }_{p a r t} \sin \theta_{P} d \theta_{P} d \phi_{P}=\left.\frac{2}{\gamma^{2}} \int_{0}^{\gamma} d t \int_{0}^{2 \pi} d \phi_{P} \frac{d^{2} W}{d \Omega d \omega}\right|_{p a r t} \frac{t}{\sqrt{1-t^{2} / \gamma^{2}}}
$$

Substituting from Equation (48) and integrating over the trigonometric functions we find

$$
\int d \phi_{P} V_{m, n}=\pi\left[\left[I_{m}^{\prime}\left(\frac{u b_{s}}{\beta}\right)\right]^{2}+\left(\frac{m \beta}{u b_{s}}\right)^{2} I_{m}^{2}\left(\frac{u b_{s}}{\beta}\right)\right]\left(\delta_{m, n}+(-1)^{m} \delta_{m,-n}\right) U_{m, m}
$$

where $\delta_{m, n}$ is the Kronecker delta and we have used $U_{m,-m}=(-1)^{m} U_{m, m}$. Note that the dependence on $\chi$ has disappeared after this integration over $\phi_{P}$. After some simplifications we find

$$
\left.\frac{d W}{d \omega}\right|_{p a r t}\left(g, u, b_{s}\right)=\frac{q^{2}}{\pi v}\left[\bar{U}_{00} I_{1}^{2}+4 \sum_{m} \bar{U}_{m, m}\left(I_{m}^{\prime 2}+\left(\frac{m \beta}{u b_{s}}\right)^{2} I_{m}^{2}\right)\right]
$$



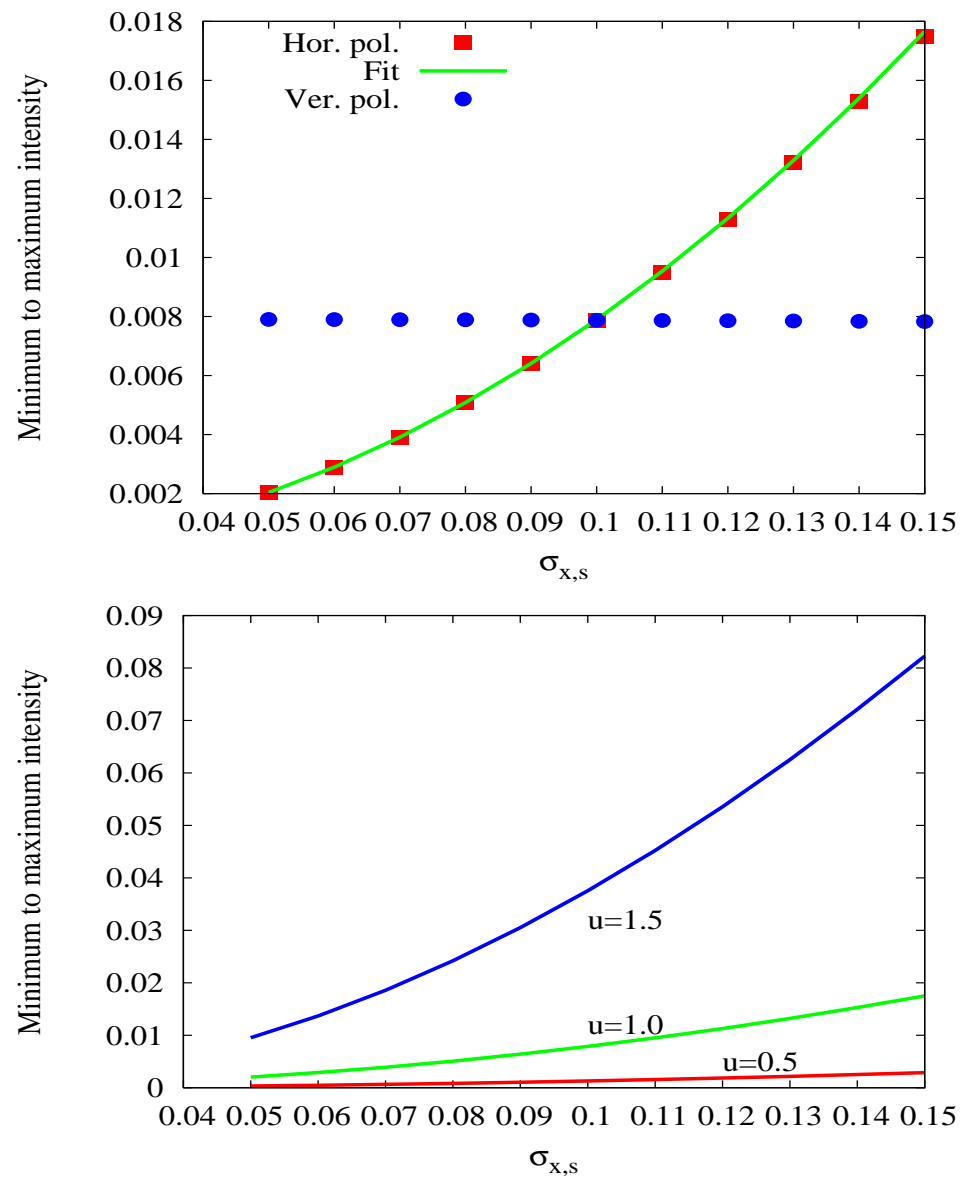

Figure 10: Top: The ratio of the minimum/maximum horizontal polarization intensity and vertical polarization intensity vs the scaled horizontal beam size $\sigma_{x, s}$ at constant $u=1, g=1.3, \sigma_{y, s}=$ $0.1, x_{0, s}=0.01=y_{0, s}$. The horizontal polarization intensity increases quadratically with the beam size as shown by the quadratic fit while the vertical polarization intensity is insensitive to the horizontal beam size changes. Bottom: The minimum to maximum of the horizontal polarization intensity for two values of $u: 0.5,1$. At $u=0.5$, the ratio is smaller which shows that the minimum intensity is less sensitive to the beam size at the lower frequency. However the ratio still increases quadratically with the beam size. 

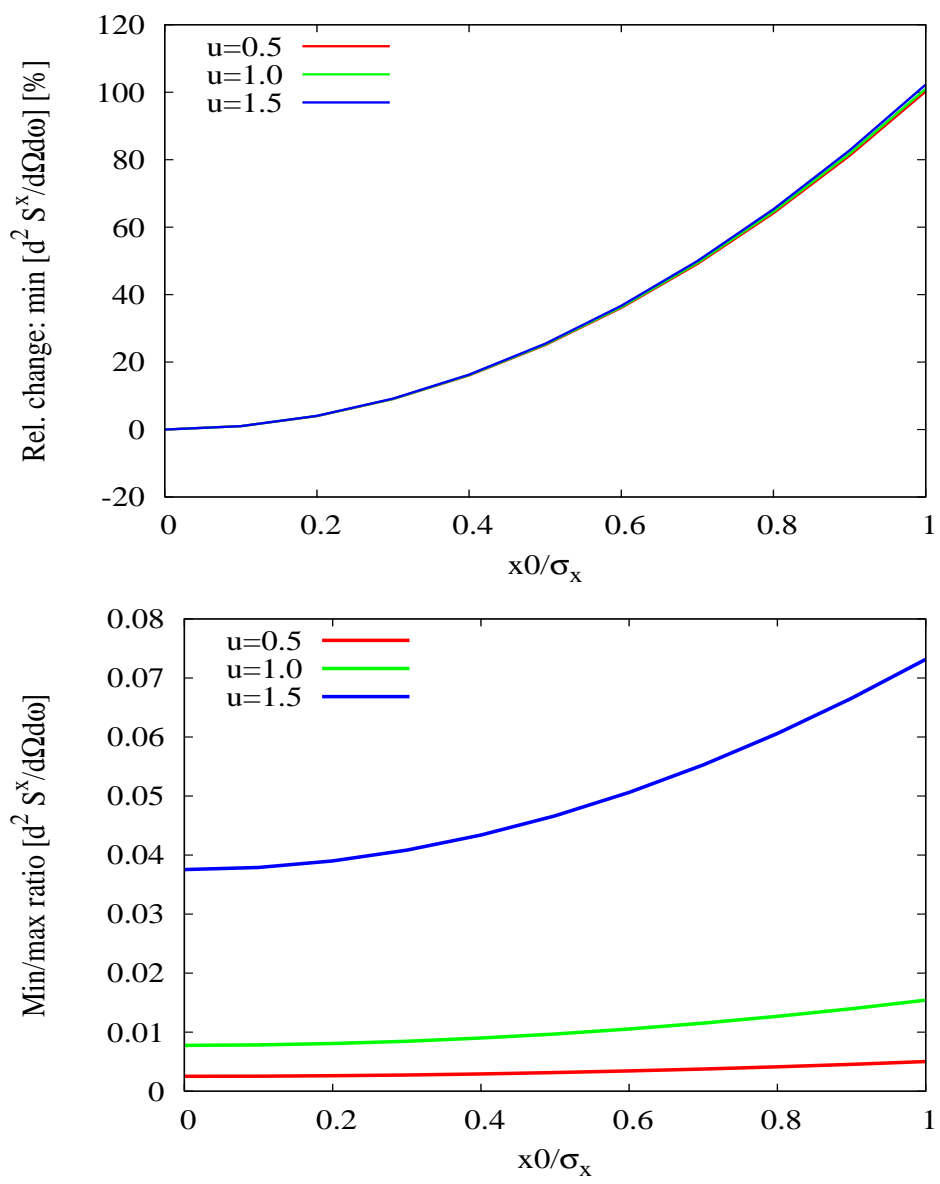

Figure 11: Top: The change in the minimum of the horizontal polarization intensity in $\%$ as a function of the the horizontal beam offset, shown in fractions of the beam size for three values of $u$. This change is not sensitive to the frequencies in this range. Bottom: Ratio of the minimum to the maximum horizontal polarization vs the offset for the same values of $u$. This ratio increases with the frequencies. 
where we have defined the integrated functions

$$
\bar{U}_{m, m}(g, u, \gamma)=\int_{0}^{\gamma} d t \frac{t}{\sqrt{1-t^{2} / \gamma^{2}}} \frac{1}{\left(1+t^{2}\right)^{2}} U_{m, m}(g, u, t)
$$

The differential spectrum from a bunch is found from

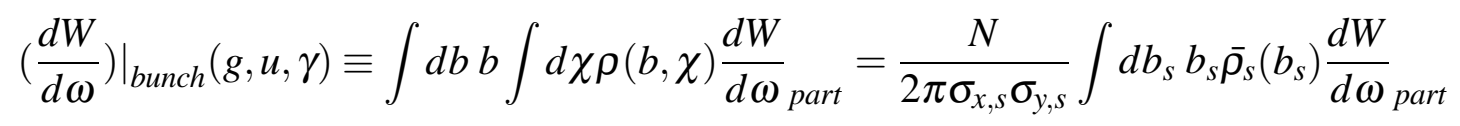

where we have integrated over the scaled density

$$
\bar{\rho}_{s}\left(b_{s}\right)=\int d \chi \rho_{s}\left(b_{s}, \chi\right)
$$

From the expression for the density in Equation (59) it follows that we can write

$$
\begin{aligned}
\rho_{s}\left(b_{s}, \chi\right)= & \exp \left[-\frac{1}{2}\left(\frac{x_{0, s}^{2}}{\sigma_{x, s}^{2}}+\frac{y_{0, s}^{2}}{\sigma_{y, s}^{2}}\right)\right] \exp \left[-b_{s}^{2} \sigma_{+, s}\right] \exp \left[-b_{s}^{2} \sigma_{-, s} \cos 2 \chi\right] \\
& \times \exp \left[\frac{b_{s} x_{0, s}}{\sigma_{x, s}^{2}} \cos \chi\right] \exp \left[\frac{b_{s} y_{0, s}}{\sigma_{x, s}^{2}} \sin \chi\right]
\end{aligned}
$$

Here we have defined the scaled beam parameters

$$
\sigma_{ \pm, s}=\frac{1}{2}\left[\frac{1}{\sigma_{x, s}^{2}} \pm \frac{1}{\sigma_{y, s}^{2}}\right]
$$

Expanding the exponentials of the trigonometric terms in Bessel functions, we have

$$
\begin{aligned}
\exp \left[-b_{s}^{2} \sigma_{-, s} \cos 2 \chi+\frac{b_{s} x_{0, s}}{\sigma_{x, s}^{2}} \cos \chi+\frac{b_{s} y_{0, s}}{\sigma_{x, s}^{2}} \sin \chi\right]= & \sum_{j \geq 0} \sum_{k \geq 0} \sum_{l \geq 0}(-1)^{j} D_{j} D_{k} D_{l} I_{j}\left(b_{s}^{2} \sigma_{-, s}\right) I_{k}\left(\frac{b_{s} x_{0, s}}{\sigma_{x, s}^{2}}\right) \\
& \times I_{l}\left(\frac{b_{s} y_{0, s}}{\sigma_{y, s}^{2}}\right) \cos 2 j \chi \cos k \chi \cos [l(\pi / 2-\chi)]
\end{aligned}
$$

where

$$
D_{j}=1 \text { for } j=0, \quad D_{j}=2 \text { for } j \geq 1
$$

After integrating over the angle $\chi$, it follows that

$$
\begin{aligned}
\bar{\rho}_{s}\left(b_{s}\right)= & \frac{\pi}{2} \exp \left[-\frac{1}{2}\left(\frac{x_{0, s}^{2}}{\sigma_{x, s}^{2}}+\frac{y_{0, s}^{2}}{\sigma_{y, s}^{2}}\right)\right] G\left(b_{s}\right) \\
G\left(b_{s}\right)= & \exp \left[-b_{s}^{2} \sigma_{+, s}\right]\left[4 I_{0}^{2}\left(b_{s}^{2} \sigma_{-, s}\right) I_{0}\left(\frac{b_{s} x_{0, s}}{\sigma_{x, s}^{2}}\right) I_{0}\left(\frac{b_{s} y_{0, s}}{\sigma_{y, s}^{2}}\right)\right. \\
& +\sum_{N o t[j=0=l]} \sum_{j \geq 0}(-1)^{j+l} D_{j} D_{2 l} I_{j}\left(b_{s}^{2} \sigma_{-, s}\right) I_{2 l}\left(\frac{b_{s} y_{0, s}}{\sigma_{y, s}^{2}}\right)\left\{D_{2|j-l|} I_{2|j-l|}\left(\frac{b_{s} x_{0, s}}{\sigma_{x, s}^{2}}\right)\right. \\
& \left.\left.+D_{2(j+l)} I_{2(j+l)}\left(\frac{b_{s} x_{0, s}}{\sigma_{x, s}^{2}}\right)\right\}\right]
\end{aligned}
$$


If the offsets vanish, i.e. $x_{0}=0=y_{0}$, this simplifies to

$$
\lim _{x_{0}, y_{0} \rightarrow 0} \bar{\rho}_{s}=2 \pi \exp \left[-b_{s}^{2} \sigma_{+, s}\right] I_{0}\left(b_{s}^{2} \sigma_{-, s}\right)
$$

Define the following function obtained after integrating over $b_{s}$

$$
F_{m}\left(u ; \sigma_{x, s}, \sigma_{y, s}, x_{0, s}, y_{0, s}\right)=\int_{0}^{1} d b_{s} b_{s} G\left(b_{s}\right)\left[\left(I_{m}^{\prime}\left(\frac{u b_{s}}{\beta}\right)\right)^{2}+\left(\frac{m \beta}{u b_{s}}\right)^{2} I_{m}^{2}\left(\frac{u b_{s}}{\beta}\right)\right]
$$

In the limit that the offsets vanish, this simplifies to

$$
\lim _{x_{0}, y_{0} \rightarrow 0} F_{m}=4 \int_{0}^{1} d b_{s} b_{s} \exp \left[-b_{s}^{2} \sigma_{+, s}\right] I_{0}^{2}\left(b_{s}^{2} \sigma_{-, s}\right)\left[\left(I_{m}^{\prime}\left(\frac{u b_{s}}{\beta}\right)\right)^{2}+\left(\frac{m \beta}{u b_{s}}\right)^{2} I_{m}^{2}\left(\frac{u b_{s}}{\beta}\right)\right]
$$

The differential spectrum from a bunch is obtained from

$$
\left.\left(\frac{d W}{d \omega}\right)\right|_{\text {bunch }}=\frac{q^{2}}{2 \pi \nu} \frac{N}{\sigma_{x, s} \sigma_{y, s}} \exp \left[-\frac{1}{2}\left(\frac{x_{0, s}^{2}}{\sigma_{x, s}^{2}}+\frac{y_{0, s}^{2}}{\sigma_{y, s}^{2}}\right)\right]\left\{\bar{U}_{0,0} F_{0}+4 \sum_{m \geq 1} \bar{U}_{m, m} F_{m}\right\}
$$

The photon yield into a bandwidth $\Delta \omega$ is

$$
\Delta N_{p h}=\frac{\alpha_{f}}{2 \pi \beta} \frac{N}{\sigma_{x, s} \sigma_{y, s}} \exp \left[-\frac{1}{2}\left(\frac{x_{0, s}^{2}}{\sigma_{x, s}^{2}}+\frac{y_{0, s}^{2}}{\sigma_{y, s}^{2}}\right)\right]\left\{\bar{U}_{0,0} F_{0}+4 \sum_{m \geq 1} \bar{U}_{m, m} F_{m}\right\} \frac{\Delta \omega}{\omega}
$$

This is the photon yield from a single bunch per turn over the full $4 \pi$ solid angle, the number of photons intercepted by the detector will be reduced by the acceptance of the detector.

Figure 12 shows the photon yield (plotted on a logarithmic scale) from a single bunch per turn into a $1 \%$ bandwidth as a function of the scaled frequency $u=\omega / \omega_{c}$ for three values of the scaled beam size $\sigma_{x, s}$. We have set the offsets $x_{0}, y_{0}$ to zero for this calculation. The photon count is about $10^{6}$ photons per bunch per turn at $u=1$ which should yield a detectable signal. The dependence of the photon yield on frequency can be fit to exponential curves with two different exponents below and above $u=1$. For example, for $\sigma_{x, s}=0.125$ we find that

$$
\begin{aligned}
\Delta N_{p h} \sim \exp [-1.28 u], & u<1 \\
& \sim \exp [-1.82 u], \quad u \geq 1
\end{aligned}
$$

These exponents are not very sensitive to the beam size $\sigma_{x, s}$, e.g the exponents below and above $u=1$ are $(-1.29 u,-1.85 u)$ for $\sigma_{x, s}=0.1$.

The photon yield does not depend sensitively on the beam size at low frequencies but at higher frequencies, the sensitivity to beam size increases.

The scaled frequency $u$ can be converted to a physical frequency by making a choice of the inner radius $a_{i n}$ of the hole. If we assume $\sigma_{x, s}=0.125=\sigma_{y, s}$ or $a_{i n}=8 \sigma$ and $\sigma \approx 0.4 \mathrm{~mm}$ at the $\mathrm{C} 0$ location in the Tevatron, then the critical wavelength $\lambda_{c}$ corresponding to the critical frequency $\omega_{c}$ is $\lambda_{c}=2 \pi a_{i n} / \gamma=19 \mu \mathrm{m}$. This is in the infra-red range. Detection in the optical 


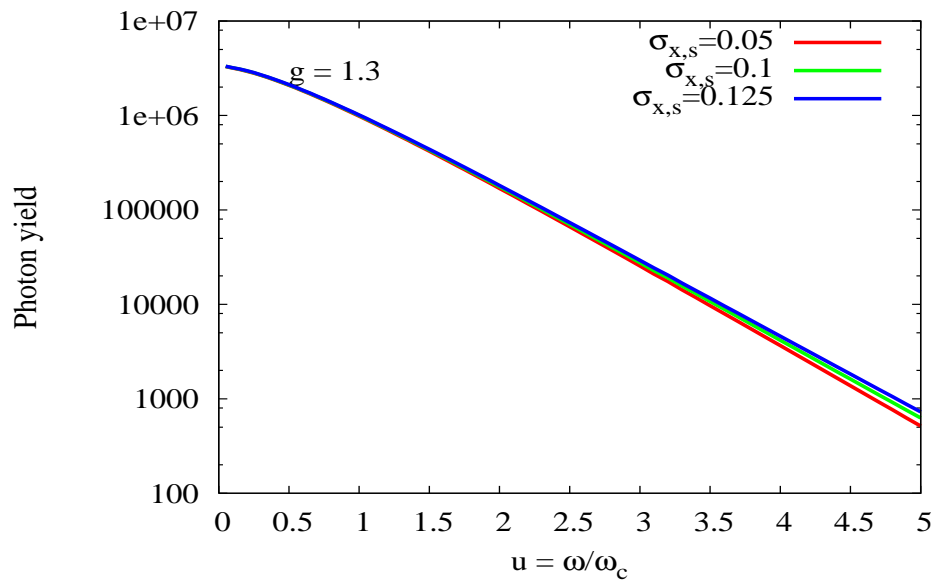

Figure 12: Photon yield (on a log scale) from a single bunch per turn into a $1 \%$ bandwidth as a function of the scaled frequency $u$ for three values of the scaled beam size $\sigma_{x, s}$. In all cases $\sigma_{y, s}=\sigma_{x, s}$. Tevatron bunch intensity and energy were used in this calculation. The photon yield is not very sensitive to the beam size at low frequencies but the relative difference in yield increases at higher frequencies.

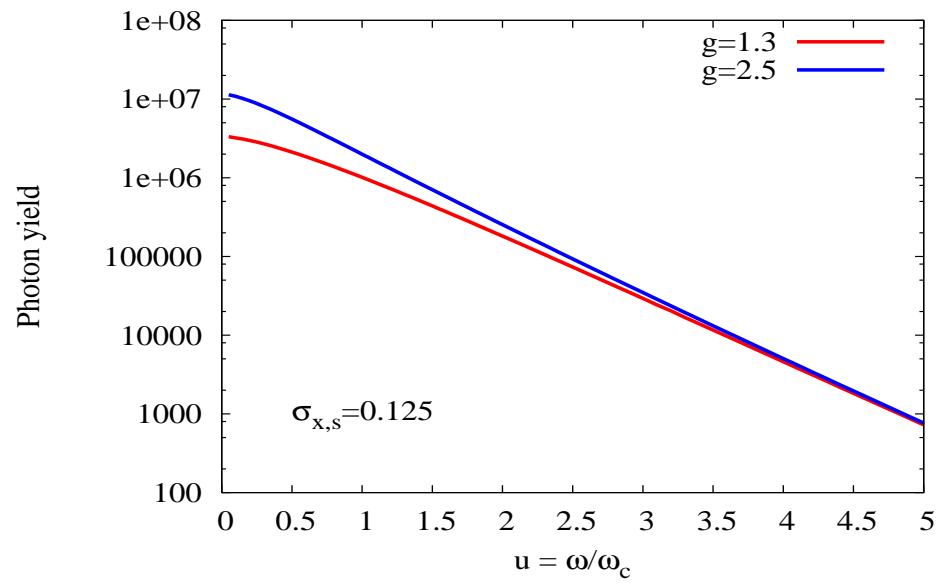

Figure 13: Photon yield (on a log scale) from a single bunch per turn into a $1 \%$ bandwidth as a function of the scaled frequency $u$ for two values of the target size $g$. Here the scaled beam size is chosen as $\sigma_{x, s}=0.125=\sigma_{y, s}$. 
range seems to be ruled out since we find that at $u=19$ or $\lambda=1 \mu \mathrm{m}$, the photon count is about $10^{-8} /$ bunch/turn or practically zero. We can increase the photon yield by increasing the target size to say $g=2.5$, the optimal value found earlier. Figure 13 shows the yields for $g=1.3,2.5$. At low frequencies $u<1.5$ the difference is significant but not so at higher frequencies. There is therefore no advantage to be gained with a larger target size if we choose to operate at frequencies above $u>2$.

We can use the photon yield to choose the frequency at which to observe the ODR. In practice the minimum photon yield will be determined by the efficiency and resolution of the camera and the level of the background synchrotron radiation which should be below the ODR photon yield. If for example we set the minimum photon yield to be $10^{4}$ photons/bunch/turn into a $1 \%$ bandwidth, then the highest scaled frequency from the plots above is $u=3.5$. For the Tevatron, this implies that observable wavelength has to be at or above $5.4 \mu \mathrm{m}$. If we integrate the signal over all 36 bunches and the bandwidth is greater than $1 \%$, then the wavelength could be reduced some more. However it will be in the few microns range and not in the optical range.

\section{OTR vs ODR spectrum}

OTR is generated when the beam goes through the material of the target. The expressions for the OTR spectrum can be found from the ODR spectrum by taking the limit $a_{\text {in }} \rightarrow 0$. With OTR we cannot define a critical frequency $\omega_{c}$ and OTR observations show that there is no frequency at which the frequency peaks. This is a qualitative difference from the ODR spectrum. Similarly we cannot also define universal expressions for the OTR spectrum which depend only on dimensionless parameters.

In this section we will briefly discuss the OTR single particle spectrum as a limiting case of the ODR spectrum. First consider the single particle at the center of the OTR target. From Equation (18) it follows that the differential angular spectrum in the far-field is given by

$$
\begin{aligned}
\left.\frac{d^{2} W^{\text {OTR }}}{d \Omega d \omega}\right|_{\text {part }} & =\frac{1}{2} \beta c\left(\frac{k q \alpha}{\pi v}\right)^{2} \frac{1}{\left[\bar{k}^{2}+\alpha^{2}\right]^{2}} T(a ; \bar{k})^{2} \\
& =\frac{1}{2} \frac{q^{2} \gamma^{2}}{\pi^{2} v} \frac{a^{2}}{\left(1+t^{2}\right)^{2}}\left[\bar{k} J_{0}(\bar{k} a) K_{1}(\alpha a)+\alpha J_{1}(\bar{k} a) K_{0}(\alpha a)\right]^{2}
\end{aligned}
$$

Here $a$ is the radius of the target, $\bar{k}=k \sin \theta_{P}, \alpha=k / \gamma$.

Figure 14 shows the dependence of the single particle OTR and ODR spectrum on the angular variable $t$. For the parameters chosen here $\left(a_{\text {out }}=1.3 a_{i n}, u=1\right)$, the maximum OTR intensity is about 8 times larger than the ODR intensity. Of this, a factor of 2.45 is due to the different areas of the material in the target for OTR and ODR. Furthermore, the OTR spectrum peaks at a smaller angle $t \approx 1 \Rightarrow \theta_{P} \approx 1 / \gamma$ while the ODR spectrum peaks at $t \approx 1.6$. This figure shows that ODR is beamed in a broader cone and at a larger angle than OTR. For the

single particle spectrum with the particle offset from the center, Equation (51) is still applicable 


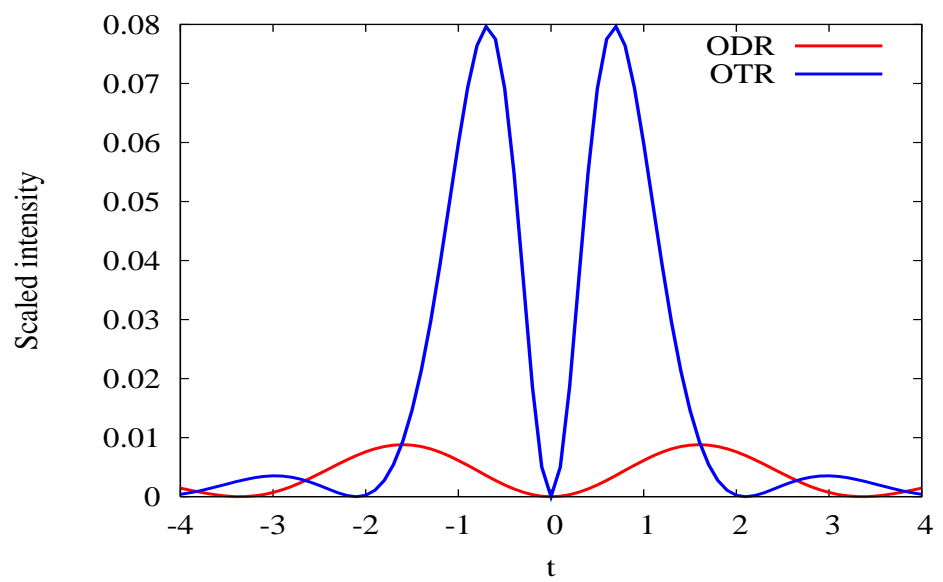

Figure 14: Comparison of ODR and OTR intensities for a single particle centered on the target. The outer radius $a_{\text {out }}$ is assumed to be the same in both cases.

for the differential angular spectrum except that $U_{m n}$ defined in Equation (48) simplifies to

$$
\begin{aligned}
U_{m, n}= & a^{2}\left[\bar{k} J_{m-1}(\bar{k} a) K_{m}(\alpha a)+\alpha J_{m}(\bar{k} a) K_{m-1}(\alpha a)\right] \\
& \times\left[\bar{k} J_{n-1}(\bar{k} a) K_{n}(\alpha a)+\alpha J_{n}(\bar{k} a) K_{n-1}(\alpha a)\right]
\end{aligned}
$$

The OTR bunch spectrum can be similarly found by taking the limit $a_{\text {in }} \rightarrow 0$ in the appropriate expressions above.

\section{Experimental Issues}

The devices and experimental conditions needed to observe ODR in the Tevatron requires a separate detailed discussion. Here we will only mention some of the issues. Synchrotron radiation from the upstream dipoles hitting the target is an important source of background and needs to be mitigated. Preliminary calculations [8] show that the level of background at a target near the $\mathrm{C} 0$ point in the Tevatron is less than the anticipated ODR flux. A mask placed upstream of the target may help to reduce this background to acceptable levels. The wavelength at which to observe the ODR depends on several competing factors. At longer wavelengths the ODR flux is higher but far infra-red detection is less sensitive, slower and complicated by other matters such as choice of windows which are sufficiently transparent at longer wavelengths. The synchrotron radiation background also increases at longer wavelengths. A satisfactory compromise might be in the vicinity of $5 \mu \mathrm{m}$. Given the likely speed with which the ODR images will be acquired, it is unlikely that bunch by bunch and turn by turn imaging will be possible with the ODR monitor. Averaging over turns will most likely be necessary but it should be possible to update images on the time scale of seconds. Calibration of the ODR measurements requires measurements by other beam imaging devices nearby. At $\mathrm{C} 0$, the synchrotron light monitor is relatively close and would be suitable for calibration of the ODR monitor. If a circular target is used, it would likely be made of two semi-circular halves which will be moved in towards 
the beam on separate stepper motors. These two halves will need to be precisely aligned and their positions measured with respect to the beam. It is very likely that due to the nature of the helical orbits, the target in the Tevatron will only be suitable for one beam. We would choose the proton beam for ease of availability and greater intensity. It would be preferable but not essential to choose the longitudinal location of the target so that both beams are not present simultaneously to avoid any effects from their parasitic interaction. Independently of this, the circular target may not be suitable if the helical orbits are separated by several beam sizes at the target. In this case the desired beam may have the required separation from the target but the opposing beam will not be far enough from the target. This could be avoided by having the two halves of the target separated by a gap. Instead of semi-circular foils, rectangular foils on either side may be preferable in this case.

We envisage that if initial measurements are successful, the ODR monitor could be used a passive device monitoring beam parameters and their changes during the length of a store. Here other operational challenges will arise. For example, beam motion which changes the position relative to the target will need to be included in the automated ODR measurement. Wake fields due to the target and heating of the target by beam induced fields are likely to be negligible but need to be considered. Some of these same issues have arisen and been resolved with the use of the pick-off mirrors for the synchrotron light mirror and will benefit from that experience. A detailed account of these and other relevant issues will appear elsewhere.

\section{Conclusions}

Our main concern here was the far field ODR spectrum from a round hole in a collider and specifically the Tevatron. We list the main conclusions

- Existence of a critical frequency and universal curves.

There is a critical frequency $\omega_{c}$ associated with ODR at which the angular spectrum intensity peaks. There is no such frequency with OTR This is a well known phenomena. However we have also shown, something not previously recognized, that the spectrum for a round hole depends only on dimensionless parameters. Hence the results seen in Figures 7 to 11 , especially the sensitivities, are universally applicable to all machines when these dimensionless parameters have the same values.

- Sensitivity to beam sizes.

The ratio of the minimum to maximum intensity is very sensitive to the beam size as seen in Figure 10. The sensitivity increases with observation frequency. The horizontal polarization is sensitive to the horizontal beam size and similarly for the vertical plane. This differs from the dependence with rectangular slits and straight edges.

- Sensitivity to beam offsets

We found that the minimum of the angular spectrum along the direction of specular reflection is most sensitive to changes in the beam offset. The ratio of the minimum to the 
maximum can also be used, this has the added advantage of higher sensitivity at increasing frequencies. Again, we found very good sensitivity (seen in Figure 11) to changes in beam offset of fractions of a beam size.

- Photon yield.

We calculated the photon yield from a single Tevatron bunch at $980 \mathrm{GeV}$. The photon yield decreases exponentially fast with frequency. Assuming an $8 \sigma$ separation between the beam and the target, the critical wavelength is $19 \mu \mathrm{m}$. The calculation predicts that photon yields of $\approx 10^{4}$ photons/bunch/turn into a $1 \%$ bandwidth will be obtained at about a $5 \mu \mathrm{m}$ wavelength. Detection at optical frequencies does not seem feasible. It is clear that the lower wavelength limit for observable ODR signals in the far-field is at a few microns, in the infra-red regime.

In this paper we did not consider the near-field spectrum in much detail except briefly in Section 3.1.2. That discussion as applicable to the spectrum from a bunch and a detailed discussion of experimental details will appear separately

\section{Acknowledgments}

I thank Alex Lumpkin, Vic Scarpine, Randy Thurman-Keup and Manfred Wendt for several useful discussions and for sharing their results with me.

\section{References}

[1] P. Karataev et al, Phys. Rev. Lett. 93, 244802 (2004)

[2] A.H. Lumpkin et al, Phys. Rev. ST Accel. Beams 10, 022802 (2007)

[3] E. Chiadroni et al, Proceedings of PAC 2007,pg 3982

[4] T. Sen, V. Scarpine and R. Thurman-Keup, Proceedings of PAC 2007, pg 1721

[5] M.L. Ter-Mikaelian, High Energy Electromagnetic Processes in Condensed Media, Wiley Interscience (1971)

[6] A.H. Lumpkin, V.E. Scarpine and G.R. Tassotto, Proceedings of PAC 2007, pg 4378

[7] Mathematica, Wolfram Research Inc.

[8] R. Thurman-Keup, private communication 